\title{
Hierofanía y matemática del canal Cumbemayo, Cajamarca, Perú
}

\section{Hierophany and mathematics of the Cumbemayo channel, Cajamarca, Peru}

\author{
Jaime Deza Rivasplata, Henry Castañeda Noriega ${ }^{2}$
}

\section{RESUMEN}

Describe las características geomorfológicas y las matemáticas empleadas en su trazo, así como su asociación con la iconografía que se conduce a lo largo del canal.

Fue construido en tres momentos históricos que se diferencia en cada segmento

El primer segmento es de 853 metros lineales, totalmente tallado en la roca y emplea la escuadra, el círculo con ángulos de 90 grados y siendo lo más saltante la inclinación constante de 1,5 mm por metro lineal.

El centro religioso está asociado al día 17 de diciembre.

Palabras clave: Cumbemayo. Cajamarca. divortium acuarium, frailones, laser

\section{ABSTRACT}

Describes the geomorphological characteristics and mathematics used in your stroke, as well as their association with iconography that is conducted along the channel.

It was built in three historical moments that differs in each segment.

The first segment is 853 linear meters, fully carved into the rock and uses the square, the circle with angles of 90 degrees and the most jumping being the constant inclination of $1.5 \mathrm{~mm}$ per linear meter.

The religious center is associated with the 17 th of December.

Keyword: Cumbemayo. Cajamarca. divortium acuarium, frailones, laser.

1. Antropolo, Investigador, Universidad Alas peruanas.Email: jaimedeza_@hotmail.com

2. Arqueologo, Universidad Nacional de trujillo, miembro residente del equipo de investigacion. Email: racktu@gmail.com 


\section{INTRODUCCIÓN}

\section{Leyenda de cumbemayo}

“...en ese lugar existían puquios de agua que servían para el cultivo y para el servicio doméstico, hasta que aparecieron unos hombres extraños $y$ malos que canalizaron y dañaron todo, hasta provocar la desaparición del agua. A estos hombres los cuismango los llamaron secses, por esta circunstancia, los cuismango se organizaron en cuadrillas para labrar un canal que recogiera el agua de todos los cerros circundantes, pero lo hicieron con respeto y con veneración a este líquido vital..." (En: Apuntes para la historia de Cajamarca. María Jaeger Vallejo, 2012)

Cumbemayo es un vocablo que deriva de dos voces quechuas lugareñas: cumbe ("piedras a modo de defensa") y mayu ("río"). Se dice también que proviene del cumpi, que significa "fino" y mayu, que significa "río". El nombre haría alusión a las características del río que tiene poco caudal, también se suele atribuir su origen a vocablos de la lengua culle, que es la antigua lengua de Cajamarca.

En el Parque Arqueológico y Área Intangible, reconocidos por la Ley 24047, se ubica en una reconocidos por la Ley 24047, se ubica en una
meseta de pastizales sin árboles el canal Cumbemayo, entre frailones de colinas talladas por la erosión del viento. Nace en la cumbre de la divisoria de los 0céanos Pacífico y Atlántico, y sigue un camino a veces recto y a veces zigzagueante. Cortando la roca viva, y calentándose con los rayos solares, lleva el agua cristalina por nueve kilómetros hasta las tierras de cultivo.

No se conoce su origen, pero todo indica que hace más de tres mil años, aún sin tener cerámica, al parecer, fue escenario de complejos ritos de invocación, los mismos que la antropología andina no puede decodificar todavía. Sus originales trazos no tendrían otra motivación. No de otra manera se pueden explicar las miles de horas invertidas en tallar 853 metros de roca viva para trasladar un litro de agua por segundo.
Bien pudo ser construido de otra manera. Si en realidad hubiera sido hecho con un sentido utilitario, es decir, para el riego, pudo haber tenido mayores dimensiones, pero las iniciales, en la roca viva, de 0,35 a $0,50 \mathrm{~m}$ de ancho por 0,10 a $0,30 \mathrm{~m}$ de profundidad solo obedecerían a un objetivo: ser parte de un escenario para el rito. Si a esto agregamos que cada cierto tramo aparecen llamativos petroglifos podríamos sugerir que tal seria la razón de su construcción.

\section{El problema de estudio}

Nuestro primer interés fue establecer las medidas y conocer las dimensiones y cálculos para el tendido del canal y las características de su transporte y asociación con los petroglifos que lo acompañan. Además otras interrogantes secundarias como:

¿Con qué herramientas calcularon la pendiente? ¿Cómo la proyectaron sin errores durante todo el trayecto? En la actualidad, tal precisión solo se logra con laser, pero si el bisoño tallador fallaba ¿cómo se hubiera remediado el error? No existen huellas de tales fallas, tampoco se han descubier to los pasos previos de experimentación.

\section{Objetivos del estudio}

Nuestro objetivo principal fue estudiar las características del canal y conocer las matemáticas que emplearon para ello y de ser posible comprender la relación del trazo con los petroglifos que lo acompañan.

\section{Estudios que anteceden}

No son numerosos los estudios arqueológicos en el valle de Cajamarca, aunque sí muy importantes. Los estudios científicos los inició en $1937 \mathrm{Ju}-$ lio C. Tello, que dejó un conjunto de materiales inéditos que aún no se dan a conocer. Diez años más tarde, los esposos Henry y Paule Reichlen, arqueólogos franceses, trabajaron en la región (años 1947 y 1948) y propusieron la columna estratigráfica y cultural del valle.
Georg Petersen G. publicó en 1947 "Cumbemayo: acueducto arqueológico que cruza la divisoria continental (departamento de Cajamarca, Perú)", estudio topográfico publicado en la Revista de la Universidad Nacional de Ingeniería, donde el autor presenta fotografías del canal y sostiene que:

[...] esta obra de ingeniería hidráulica cruzaba la divisoria continental, conduciendo las aguas de la vertiente del Océano Pacífico a la cuenca del río Amazonas, y, por ende, al Océano Atlántico [...] $(1947,113)$.

La publicación presenta los mapas elaborados con instrumentos topográficos de la época, y señala el trazo completo del canal desde la captación hasta el ingreso a Cajamarca.

Antonio Núñez Jiménez (1986) publicó reproducciones de algunos sitios de la región con petroglifos, con propósito informativo. Debemos recordar también los estudios y opiniones de científicos sociales como Fernando Silva Santisteban y Roger Ravínez, intelectuales cajamarquinos que ratifican los estudios anteriores.

La Corporación de Desarrollo de Cajamarca publicó en 1985 un estudio topográfico de la primera parte del canal, incluyendo 89 secciones y fotografías en cada sección; realizado con instrumentos topográficos, el estudio se limita a describir la morfología del canal. A pesar de que se ha escrito y publicado sobre el canal Cumbemayo, básicamente se repite lo escrito por Julio C. Tello sobre la base de los trabajos realizados durante la expedición arqueológica al Marañón en 1937..

En 1997, los arqueólogos Edgardo Silva Mego, César Cornelio Lecca y Alfredo Narváez participaron también trabajando en el inicio del canal y ofreciendo información arqueológica de gran valor.

En su estudio de tesis William Guillén Padilla escribe lo siguiente:
[...] Teniendo en cuenta la concepción moderna de agricultura, el trabajo habría priorizado el producto agrícola y no el hombre y su cultura (agrónomo). La dinamita habría cumplido un rol fundamental, igualmente el cemento. Prueba de ello es el canal moderno construido paralelo al prehispánico. La diferencia notable no solo radica en la forma, sino en la concepción: el canal prehispánico surca el divortium acuarium al ras de suelo, el ca nal moderno a través de un túnel, pues la cota de inicio así lo exige [...] (W. Guillén 2002:68)

La técnica agrícola se manifiesta en los sistemas de riego por secano y en los canales de riego por gravedad, en los que resulta sorprendente el manejo de la inclinación, los frenos y aceleración del caudal; pero el otro instrumento es la chaquitaclla y su complemento: la chira o sichira básicamente, acompañada del azadón, la raucana o rocuana, la jallmana, la chinca de cuerno de venado, el allachu, la huactana, el huarmicpanan, la taclla, la pacpana o cuti, el bastón de sembrar, entre otros desterronadores. Tales herramientas eran simples, adecuadas para plantas de raíces cortas, como el maíz (Zea maiz) por ejemplo que no precisaba de una roturación profunda de suelo.

Todas produjeron exitosos resultados, pero siempre dentro de un manejo social coordinado y sustentado en el ayni, basado a su vez en las norma de reciprocidad. La fuerte tradición corporativa permitio el trabajo de las comunidades organizadas voluntariamente en torno de objetivos comunes. El sistema de trabajo colectivo (minga o minka), el de reciprocidad (ayni), y la mita estatal suplieron la falta de instrumentos complejos y de mayor tamaño.

Es importante establecer que en el desarrollo de los sistemas de irrigación no se debe tomar en cuenta solo un factor, o suponer la primacía de uno o dos de ellos por sobre los demás, pues estas obras no son el resultado de un plan genera inspirado por un genio constructor, conducido $y$ ejecutado en un período corto de tiempo, o por una generación o dos. El perfeccionamiento y ex- 
tensión de los canales fue gradual, temporal y social, y se dio a medida que las familias (linajes) se expandían y necesitaban de más áreas de cultivo.

Los canales se erigieron gracias a la concurrencia de cuatro factores principales y condicionantes: geomorfológicos, climáticos, técnicos y sociales.

Las características geomorfológicas obligaron a los aldeanos a buscar respuesta a estas interrogantes básicas: ¿Por dónde debe conducirse el canal? ¿Qué tipos de suelo se deben evitar? ¿Qué relación debe tener el volumen de captación o caudal inicial con la longitud del canal, para transportar el agua necesaria y concluir adecuadamente el riego de los terrenos de cultivo? ¿Cuál es la formula correcta para lograr una eficiente conducción del agua? o, en otras palabras, ¿Cual es la fórmula que correlaciona la longitud del canal, la cantidad de agua a conducir y su pérdida por filtración y evaporación con la extensión del terreno agrícola a cultivar? Además, en este tipo de suelos, donde el promedio de pérdida de agua canalizada por kilómetro de recorrido es elevado, ¿cuál debía ser la velocidad, resistencia e impermeabilización para lograr reducir al mínimo las pérdidas del agua que traslada?

Por supuesto, para la construcción de estos canales se requería que los responsables de encontrar el curso correcto del agua poseyeran habilidades especiales. Probablemente, las técnicas de construcción se introdujeron poco a poco, según surgían los contratiempos planteados por las nuevas circunstancias. Un criterio adoptado respecto al punto de captación fue que cuanto más arriba se instalaba la bocatoma, mayor era la zona en pendiente que podía ser regada. Por ello, el punto más alto del Cumbemayo se ubicó en el cuello del valle, donde el río comienza a bajar al río Magdalena para atravesar el delta fluvial de su propio valle.

El conocimiento de estos principios básicos de riego por gravedad les permitió controlar la velocidad cuando los desniveles resultaban muy pronunciados y no se tenían espacios suficientes para extender el trazo. Esto los llevó a construir segmentos del canal con innovadas variaciones en la geometría del cauce.

El canal tiene tres segmentos que son indicadores de cambios conceptuales en relación con su utilidad, los mismos que han sido progresivos a medida que aumentaba la necesidad de nuevas áreas de cultivo y los ritos iniciales se fueron modificando, agregando un nuevo valor utilitario-

El primero, de finalidad ritual $(853 \mathrm{~m})$, está tallado totalmente en la roca viva, y es la parte mejor conservada. En su trazo, superaron los obstáculos sin destruir las rocas fuertes, que bien hubieran podido ser quitadas; sin embargo, los constructores prefirieron tallar sus orillas o hacer túneles para mantener la pendiente mínima, lo que muestra su alto dominio matemático.

El segundo tramo $(2500 \mathrm{~m})$ obedece a la exten sión del rito agrícola, y fue construido tallando roca de la falda del cerro y en parte levantando el borde paralelo artificialmente con piedras y relleno.

El tercero, hecho en zanja, a tajo abierto (5 650 $\mathrm{m}$ ), fue erigido cuando se hizo necesario conducir el agua para usos prácticos de riego, actualmente se encuentra abandonado.

\section{MÉTODOS Y MATERIALES}

\section{El estudio se desarrolló en tres fases:}

a. Revisión bibliográfica, que registra muy escasa bibliografía, sólo se encuentran publicados seis trabajos que merecen tenerse en cuenta, pues hay otros más de contenido periodístico, pero aun asi no llegan a una decena.

b. Exploración de campo y satelital.

c. Mediciones de campo con empleo de estación total Laica y GPS y por supuesto lo que es propio de la arqueología: notas, registros, bitácora, sistemáticos, fotografía y dibujo.
Finalmente trabajo de reproducciones, análisis, evaluaciones, comparaciones, sistematización de la información.

El trabajo de campo se desarrolló durante los meses de marzo a octubre de 2012 y con revisión de datos para confirmar en tres oportunidades (2014, 2017 y 2019), evitándose recoger material o restos de superficie que además de ser casi inexistente, es de factura tardía que no coresponde al canal.

\section{RESULTADOS}

Milímetro y medio de pendiente por cada metro de longitud es suficiente para comprobar el dominio de la hidráulica hace más de tres mil años. El complejo del riego canalizado se inicia con la captación del agua que se realizaba en un dique cuadrangular de $20 \mathrm{~m}$ de lado, de piedra asentada, cuyo fondo se encuentra a $3578 \mathrm{msnm}\left(7^{\circ} 11\right.$ $27,1^{\prime \prime}$ LS - 78 34' 57,4" LO). De ahí parte el canal hacia la plaza principal o ritual, donde se le une un canal de piedra de la primera quebrada colectora en el lugar denominado como "La Toma".

El inicio del canal es un verdadero complejo hidráulico que comprende, además de la toma de captación, dos elementos importantes: el llamado Altar y una plataforma sólida con dibujos tallados en la superficie de la roca, en la que destacan dos pares de pies, uno dirigiéndose al norte y el otro con dirección al sur. Además, se observa in situ la estructura necesaria para medir el caudal de ingreso del agua.

El canal tiene tres segmentos que son indicadores de cambios conceptuales en relación con su utilidad. El primero, de finalidad ritual $(853 \mathrm{~m})$, está labrado totalmente en la piedra del lugar, constituyéndose en parte natural del paisaje.

El segundo (2500 m), obedece a la extensión de censtruido tallando la roca de la falda del cerro y en parte levantando el borde paralelo artificialmente con piedras y relleno.
El tercero, hecho en zanja, a tajo abierto (5 650 $m$ ), fue erigido cuando se hizo necesario conducir el agua para usos prácticos de riego.

\section{Características del canal}

Nuestro estudio solamente comprende la primera etapa del canal, que es la más compleja tallada sobre roca y cuyas características son las siguientes:

\section{Dimensiones}

El ancho y la profundidad varían. Si bien en el ancho no existen diferencias mayores 35 a $50 \mathrm{~cm}$ la profundidad por ser excavada en roca viva, varía según las sinuosidades del terreno, para mantener constante la inclinación entre los 10 y 30 $\mathrm{cm}$ en los segmentos superficiales y de $20 \mathrm{~cm}$ a 1 metro cuando la superficie del terreno se eleva.

\section{Longitud}

La longitud del canal está en función de la ubicación y extensión del área a regar, así como de caudal considerado necesario. Tiene una longitud total de 9003 metros y consta de tres segmentos con técnicas de construcción y cronologías diferentes:

Segmento 1: $853 \mathrm{~m}$ Segmento 2: $2500 \mathrm{~m}$ Segmento 3: $5650 \mathrm{~m}$

\section{Curvas}

Dos son las figuras geométricas básicas: el círculo y el cuadrado, cuyos segmentos constituye curvas de $90^{\circ}$ para enlazar los tramos rectos diseñados en un arco de curvatura circular, trazado por el principio del compás y utilizando una cuerda, la que al girar sobre el centro de curvatura une los tramos rectos. Estas curvas cerradas se encuentran en los inicios del canal, y la presencia de esquinas en escuadra frena y acelera el caudal. 


\section{Caudal y velocidad}

Considerando las variaciones del área mojada en los dos primeros segmentos, la tirante promedio, la pendiente y rugosidad del suelo y su relación con la longitud de los mismos, podemos plantear que el tipo de flujo que lo recorre es de régimen subcrítico, uniforme. Posiblemente, dadas las condiciones descritas, este debió ser de 0,0743 $\mathrm{m} 3 / \mathrm{s}$.

El control del flujo se logró gracias a una pendiente casi rasante, que varía por sectores, a veces de 1 $\mathrm{mm}$ a $3 \mathrm{~mm}$ por metro lineal, otras se profundiza hasta los $7 \mathrm{~mm}$ por metro lineal, para alcanzar la velocidad que le permita elevarse a otros segmentos de niveles más altos. Pero en promedio, desde sus orígenes hasta el término del segundo segmento ( $3550 \mathrm{~m}$ a $3545 \mathrm{~m}$ de altitud) es de 1,6 $\mathrm{m}$ por kilómetro de longitud, lo que la da una velocidad de $0,2935 \mathrm{~m} / \mathrm{s}$. No se encuentran segmentos importantes que ofrezcan características de grandes variaciones en la velocidad.

Angostando o ampliando en algunos tramos el ancho del canal, reducían la velocidad y disipaban energía por efectos de expansión. También construyeron zigzags en el primer segmento, y algunas veces colocaron obstáculos (por lo general grandes bloques de piedra) en las orillas del cauce, con el fin expuesto.

Sabido es que cuando un canal con flujo subcrítico $(\mathrm{F}<1)$ entra en un canal más ancho, se expande y desacelera. Un canal supercrítico $(\mathrm{F}>1)$, en cambio, acelera su velocidad cuando el fondo del canal aumenta bruscamente en pendiente negativa, y el tirante o profundidad disminuye. En otras palabras, esta técnica de acelerar o frenar el flujo, que se explica con la fórmula de Froude, fue conocida y muy bien empleada por los constructores andinos.

V= Velocidad media de la sección, en $\mathrm{m} / \mathrm{s}$ $\mathrm{g}=$ Aceleración de la gravedad, en $\mathrm{m} / \mathrm{s} 2$
D = Longitud característica de la sección, en metros

$\begin{array}{ll}\text { Régimen subcrítico } & \mathrm{F}<1 \\ \text { Régimen crítico } & \mathrm{F}=1 \\ \text { Régimen supercrítico } & \mathrm{F}>1 \\ \mathrm{~F}=\text { Número de Froude } & \mathrm{F}=\frac{\mathrm{V}}{\sqrt{\mathrm{V} \mathrm{gD}}}\end{array}$

\section{Pendiente de la rasante}

La pendiente al inicio del canal es muy cercana a la horizontal, con un promedio de $0,001 \mathrm{~m}$ o,002m, para nuevamente incrementarse al término del segundo y último tramo del canal, construido a tajo abierto y que se dirige al valle con una pendiente promedio de $0,007 \mathrm{~m} / \mathrm{m}$.

Este desnivel permite que la velocidad del caudal se mantenga en un rango de 0,3 a $3 \mathrm{~m}$ por segundo $(0,3 \mathrm{~m} / \mathrm{s}<\mathrm{V}<3 \mathrm{~m} / \mathrm{s})$, ya que no puede ser menor de $0,3 \mathrm{~m} / \mathrm{s}$ porque ocasionaría sedimen tación y colmatación en los canales, y tampoco puede ser mayor a $3 \mathrm{~m} / \mathrm{s}$ porque provocaría erosión en la base del canal.

\section{Rugosidad}

En el primer segmento, el fondo es liso, de piedra tallada-William Guillén le señala su tesis $(\mathrm{Ob}$ Cit.) un coeficiente de 0,033 - nosotros hemos registrado $=0,035$ diferencias explicables. En el segundo segmento por las características del fondo, las paredes laterales verticales y lizas y su sección compacta, la resistencia debió tener un coeficiente medio, normal.

\section{Elementos del canal}

Toma o punto de captación. Es un dique cuadrangular de retención en cuya construcción intervinieron dos variables principales: la longitud o recorrido que debía tener el canal y la profun- didad del cauce del río. En este caso, es el punto de mayor altitud, donde confluyen cuatro pequeñas quebradas que alimentan de agua al canal.

Compuertas. Son En el recorrido no se observan compuertas, por lo menos en los dos primeros segmentos, cuyo trazo es limpio y visible con un medidor de agua.

Acueducto. Conducto artificial elevado que se observa en dos lugares en el segundo segmento para cruzar de faldas y depresiones no mayores de diez metros por uno de altura.

Tunel. Existe uno, prolongacion del acueducto, que cruza la falda del cerro, que tiene diez metros de largo por $0.80 \mathrm{~m}$ de alto y $0.60 \mathrm{~m}$ de ancho similar.

Tomando como base los resultados presentados los datos promedio son:

Tirante promedio (y): $0,487 \mathrm{~m}$

Ancho de solera (b): 0,520 m

Coeficiente de rugosidad: 0,03

Pendiente: $0,001 / 1,5 \mathrm{~m} / \mathrm{m}$

Caudal: $0,0743 \mathrm{~m} 3 / \mathrm{s}$

Velocidad: $0,2935 \mathrm{~m} / \mathrm{s}$

Número de Froude: 0,1343

Tipo de Flujo: Subcrítico

Los petroglifos

Por su ubicación, se han considerado: Los existentes al inicio en la gruta o Santuario, en la segunda gruta a trescientos metros al oeste, en la plaza principal, en la trayectoria de los dos primeros segmentos y en la tercera gruta ritual.

\section{La primera gruta. El Santuario.}

Se sostiene que esta gruta es un espacio de adoración, por la presencia de sus petroglifos, por su orientación al Sol y por su ubicación en el conjunto. La base es casi perfectamente circular ( 3,10 x 3,30 m con una altura de 2,75 m). Aunque el nombre no es correcto, como hemos dicho, estaría asociado al inicio del rito, al tiempo que comenzaría cuando la sombra se proyectaba al medio de la gruta, en una hornacina cuadrangular, en cuyo centro se registra el desgaste de la piedra por la escorrentía del agua que filtra de la parte superior de la colina y que corre depositándose en cinco hoyos, cuatro de los cuales nos recuerdan a la Cruz del Sur.

Es de notar que en el mes de diciembre (día 17) se proyecta la sombra al interior de la cueva, cayendo al medio de una hornacina $(70 \mathrm{~cm} \times 60$ $\mathrm{cm})$. Hemos repetido esta experiencia en tres años posteriores $(2014,2017,2019)$ y es invariable esta escena, mas no así en otros meses. ¿Se trataría del espacio y fecha ritual al inicio de las lluvias? Consideramos oportuno comentar que tal vez esta sería su función.

Existen otros hoyos rituales, cuatro de ellos imitando a la Cruz del Sur, cuyo brazo izquierdo coincide con el centro de la hornacina.

Una barrera de ingreso de $0,20 \mathrm{~m}$ de alto muestra figuras en ambas caras, también el piso y las paredes del interior. Cruces, caras, las tres escalinatas, plantas, animales (culebras), figuras humanas en movimiento, otras irreconocibles, cubren la gruta en su totalidad.

Querer interpretar su lectura hoy no es posible ello convoca a un equipo de especialistas, porque aun el símbolo no parece estar asociado a un sonido cuyo conjunto forme palabras.

\section{La segunda gruta. "Santuario menor"}

Se ubica a $300 \mathrm{~m}$ al oeste de la anterior, a 3592 metros de altitud y a $7^{\circ} 11^{\prime} 19,8^{\prime}$ LS / $78^{\circ} 34^{\prime} 33,3^{\prime}$ 
O). Parecería que por sus características de ubicación, dimensiones, como el estado actual de las figuras, y por estar próxima y asociada a un camino angosto $(0,40 \mathrm{~m} \times 5 \mathrm{~m}$ de largo) tallado en la piedra que cruza el abra, a 3620 metros de altitud ( $7^{\circ} 11^{\prime} 27,1^{\prime \prime}$ LS / $78^{\circ} 34^{`} 57,4^{\prime \prime}$ LO) y con finalidad ritual, es anterior al santuario. $\mathrm{Al}$ ingreso, en la boca, se encuentra un monolito con figuras distintas a las anteriores, bastante erosionadas y más simples.

De él parten canalitos que repiten el trayecto de la escorrentía cuando llueve. Se encuentra sobre una falda que excavada nos daría grandes sorpreas.

\section{La plaza principal}

Es el complejo hidráulico religioso por excelencia. Se inicia en una gran plaza que descansa sobre una plataforma donde se juntan el canal que viene de la toma en el divortium acuarium con otro menor de piedra, en lo que se conoce como la bocatoma de entrada, con una piedra con marcas horizontales que recuerda la mira graduada que mide el caudal de ingreso.

Aquí se observan tres bloques, a manera de escalinatas, en los que se han tallado las primeras figuras, y constituyen el denominado altar o púlpito.

A cinco metros destaca la presencia de una plataforma de 1,80 m x 2,6 m, en cuya superficie encontramos, asociados a una doble cadena de hocontramos, asociados a una doble cadena de ho-
yos pequeños, dos pares de plantas de sandalias. El primer par de $11 \times 23 \mathrm{~cm}$, que inicia el camino al norte, y el segundo de $9,5 \times 22 \mathrm{~cm}$, que retorna con dirección al sur. Los guías atribuyen estas figuras a supuestos matrimonios allí suscitados.

Cerca se encuentra una roca circular con figuras muy erosionadas, destacando el espiral, a orillas del canal. La parte o cara superior es plana y presenta figuras que por la naturaleza de la piedra están casi borradas. Su presencia hablaría del movimiento, energía, seguimiento, conducción, vida, etc. Este símbolo se repite en todas las culturas y se le califica como la culebra, el cosmos. En la cultura Nasca, se le encuentra en la cola de El Mono.

\section{La tercera gruta}

Parece que pertenece a la siguiente extensión del canal, aprovechando una gruta natural a 3553 metros de altitud ( $7^{\circ} 11^{\prime} 31,6^{\prime \prime}$ LS / $78^{\circ} 34^{\prime} 35,7^{\prime}$ LO). Se encuentra a un costado del trayecto, junto a un pequeño acueducto del canal que se prolonga cortando la roca viva y en cuyos interiores se observan dos manos de tamaño natural y pies pequeños.

El ingreso se facilita por una gradería tallada desde la parte inferior hasta la boca de la gruta. Sus figuras son más reconocibles, sobresaliendo el símbolo de las tres gradas acompañado por la cruz cuadrada.

A partir de este lugar, se registran en las paredes de piedra del canal figuras aisladas cada cierto tramo, como caras, brazos doblados en escuadra mostrando la palma de la mano, caras de frente y sobre todo cruces cuadradas sin asociación a otras figuras.

$\mathrm{Al}$ término es visible un mural de tres metros de largo, como una cinta de inconfundible inscrip ción conceptual. Entonces, el canal se profundiza en la tierra via a Layzon.

\section{En el trayecto}

Destacan algunos trazos de tipo Chavín, motivo por el que se le asocia con esta cultura, lo que es posible, pero influenciando o contribuyendo a su formación. Todas las figuras están relacionadas con el canal; no de otra manera se explica su ubicación, para expresar ideas asociadas al trayecto del agua.

Las figuras se encuentran aisladas, separadas po cierta distancia, o en murales, limitadas por el tamaño de la piedra, pero constituyendo un con- junto. Cabe destacar la presencia de la cruz cuadrada, de la cual evoluciona la chacana. Este símbolo es la base de los trazos en el canal. También se pueden ver rostros o partes del cuerpo, como manos, pies, brazos en alto, caras, ojos, espirales, escalinatas, surcos, hoyos.

Importante es la presencia de la Cruz del Sur, ligada a las lluvias, su presencia no ha dejado de tener vigencia. Muchas costumbres y tradiciones de Cajamarca están asociadas a las cruces: fiestas religiosas, ubicadas sobre los techos para protección, etc.

Asociado a las cruces se tiene un petroglifo en forma de estrella de cuatro lados, con resplandor con cuatro líneas a cada lado. La "estrella" más resplandeciente en el firmamento es, sin duda, Venus.

\section{DISCUSIÓN}

Considerando la magnitud del canal de Cumbemayo, así como sus resultados, estas variables debieron ser conocidas por sus constructores previamente al tendido; no de otra forma nos explicamos la geometría del canal y la perfección de su talla en la roca granítica, sus dimensiones constantes, medidas topográficas y métodos de control hidráulico utilizados, los mismos que les permitieron alcanzar una eficiente conducción del agua; sin embargo, lo más sorprendente ha sido descubrir que la inclinación del canal mantuvo una relación constante de uno a dos milímetros por metro de longitud, lo que evidencia el desarrollo de una ingeniería de gran nivel y un dominio total del tallado de la piedra.

El elemento básico de toda obra de riego es, sin duda, la observación del lugar donde se va a ubicar la obra hidráulica. Este método empírico dio como resultado que el canal de Cumbemayo haya sido ubicado en el lugar preciso, a la altura de la divisoria de aguas, donde existen condiciones para rendirle culto, sin olvidar el hecho de estar asociado a un elemento primordial: la piedra.
La derivación de aguas de una vertiente a otra (de un océano a otro), señalada por Petersen en 1947 es una propuesta tal vez exagerada, pues para eso debieron tener una información tal como la que poseemos actualmente. Lo cierto es que supieron canalizar y derivar parte de las aguas que iban la costa para beneficio de la sierra.

Tirante promedio (y): 0,487 $\mathrm{m}$

Ancho de solera (b): 0,520 m

Coeficiente de rugosidad: 0,03

Pendiente: $0,001 / 1,5 \mathrm{~m} / \mathrm{m}$

Caudal: $0,0743 \mathrm{~m} 3 / \mathrm{s}$

Velocidad: $0,2935 \mathrm{~m} / \mathrm{s}$

Número de Froude: 0,1343

Tipo de Flujo: Subcrítico

\section{CONCLUSIONES}

A juzgar por estos resultados, el escaso caudal de este canal no habría tenido fines agrícolas de abastecimiento para grandes poblaciones; más bien habría servido para regar pequeñas áreas, y por la naturaleza de la sociedad de esa época habría constituido parte de un complejo ritual de riego y espacios de siembra: toda una concepción mágica del proceso de fecundación de la tierra la llegada de los frutos en un espacio abierto.

Es de notar que en el mes de diciembre (día 17) se proyecta la sombra al interior de la cueva, $\mathrm{ca}$ $\mathrm{cm}$ ).

Hemos repetido esta experiencia en años posteriores $(2014,201772019)$ en la misma fecha y es invariable esta escena, mas no así en otros meses. ¿Se trataría del espacio y fecha ritual al inicio de las lluvias? Consideramos oportuno comentar que tal vez esta sería su función. 
Existen otros hoyos rituales, cuatro de ellos imitando a la Cruz del Sur, cuyo brazo izquierdo coincide con el centro de la hornacina.

\section{REFERENCIAS BIBLIOGRÁFICAS}

Corporación de Desarrollo de Cajamarca

Un estudio topográfico de la primera parte del canal, incluye 89 secciones y fotografías en cada sección

Deza Rivasplata, Jaime

2010

El agua de los Incas. Fondo editorial de la Universidad Alas Peruanas, Lima.

2012

Cumbemayo. Fondo editorial de la Universidad Alas Peruanas, Lima.

Guillén Padilla, William

2002

Tesis de licenciatura. UTC. Cajamarca

Núñez Jiménez, Antonio

1986

Publicó reproducciones de algunos sitios de la región con petroglifos, con propósito informativo

Petersen, Georg, G.

1947

Cumbemayo: acueducto arqueológico que cruza la divisoria continental (departamento de Cajamar-

ca, Perú)", Revista de la Universidad Nacional de Ingeniería, Lima.

\section{Reichlen, Henry y Paule}

$1947-48$

Columna estratigráfica y cultural del valle Cajamarca
Figura $\mathrm{N}^{\circ}$ 1.Los frailones de Cumbemayo, donde se inicia el proceso ritual de los canales. Obsérvese el primer altar.

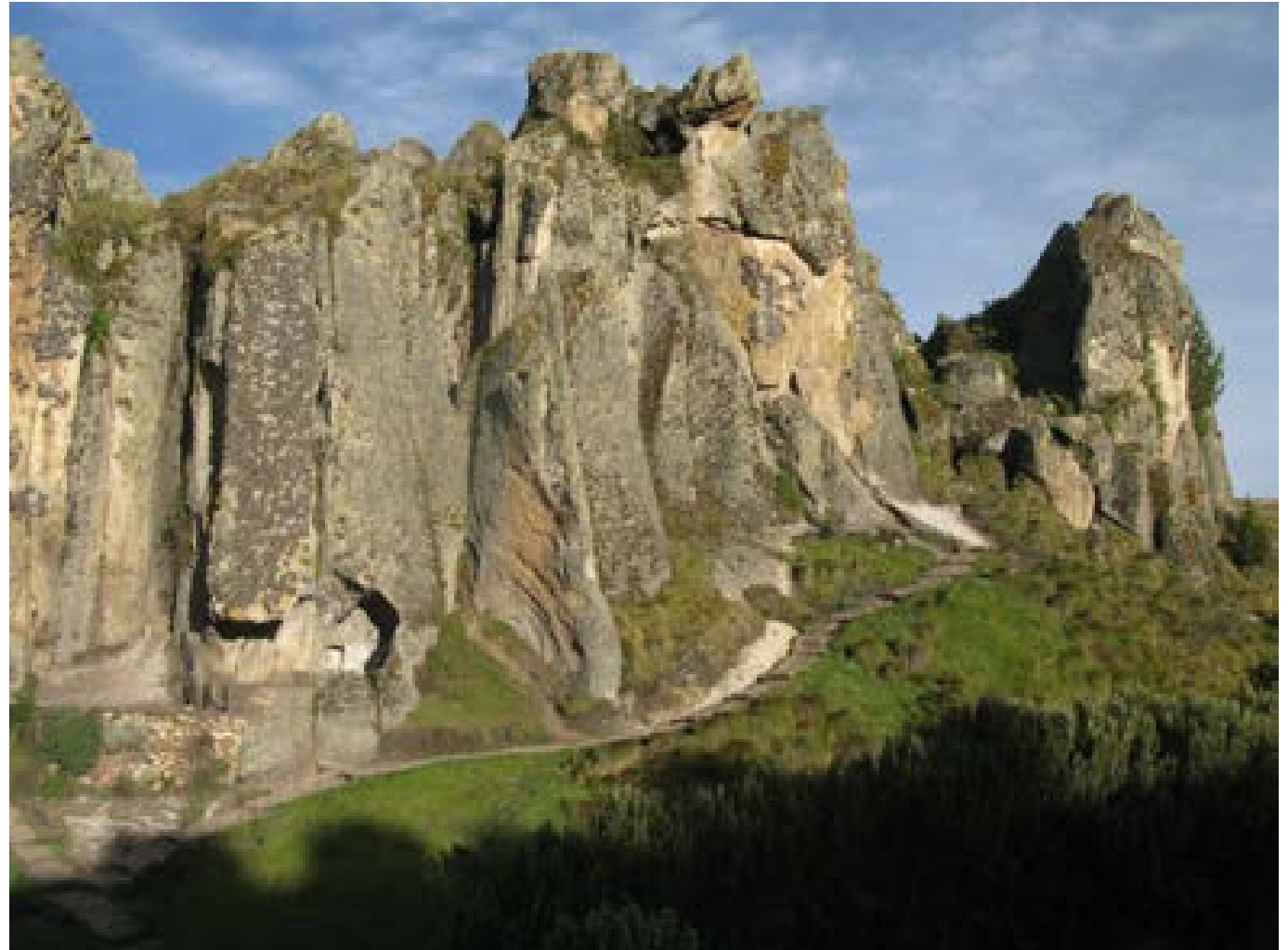

Figura $\mathrm{N}^{\circ}$ 2. El primer altar en la falda de los frailones con figuras de una hirofanía predecesora de la cultura Chavín.

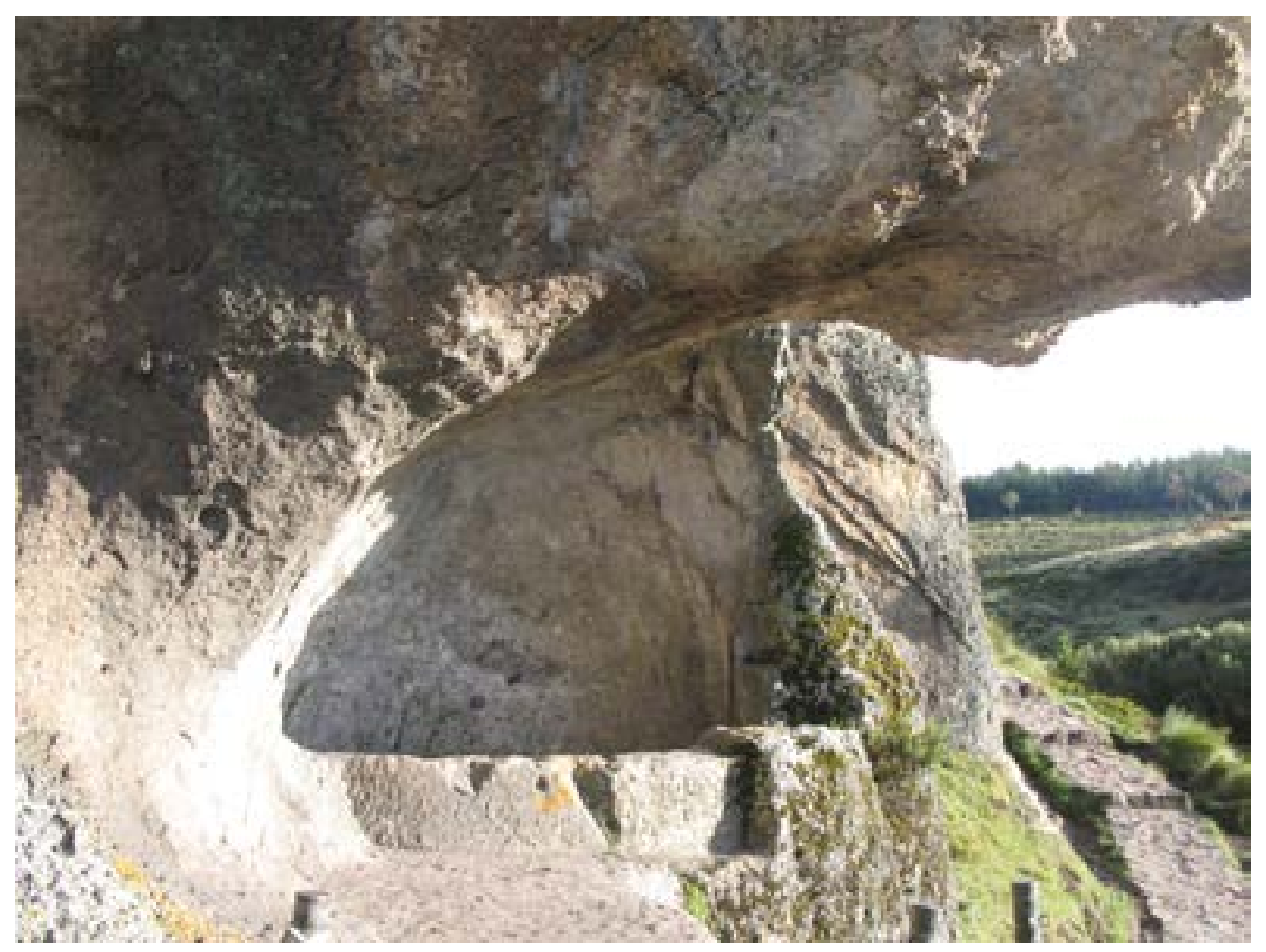


Figura $\mathrm{N}^{\circ}$ 3. Proyección de la sombra en la hornacina al centro del altar, los dias 17 de diciembre.

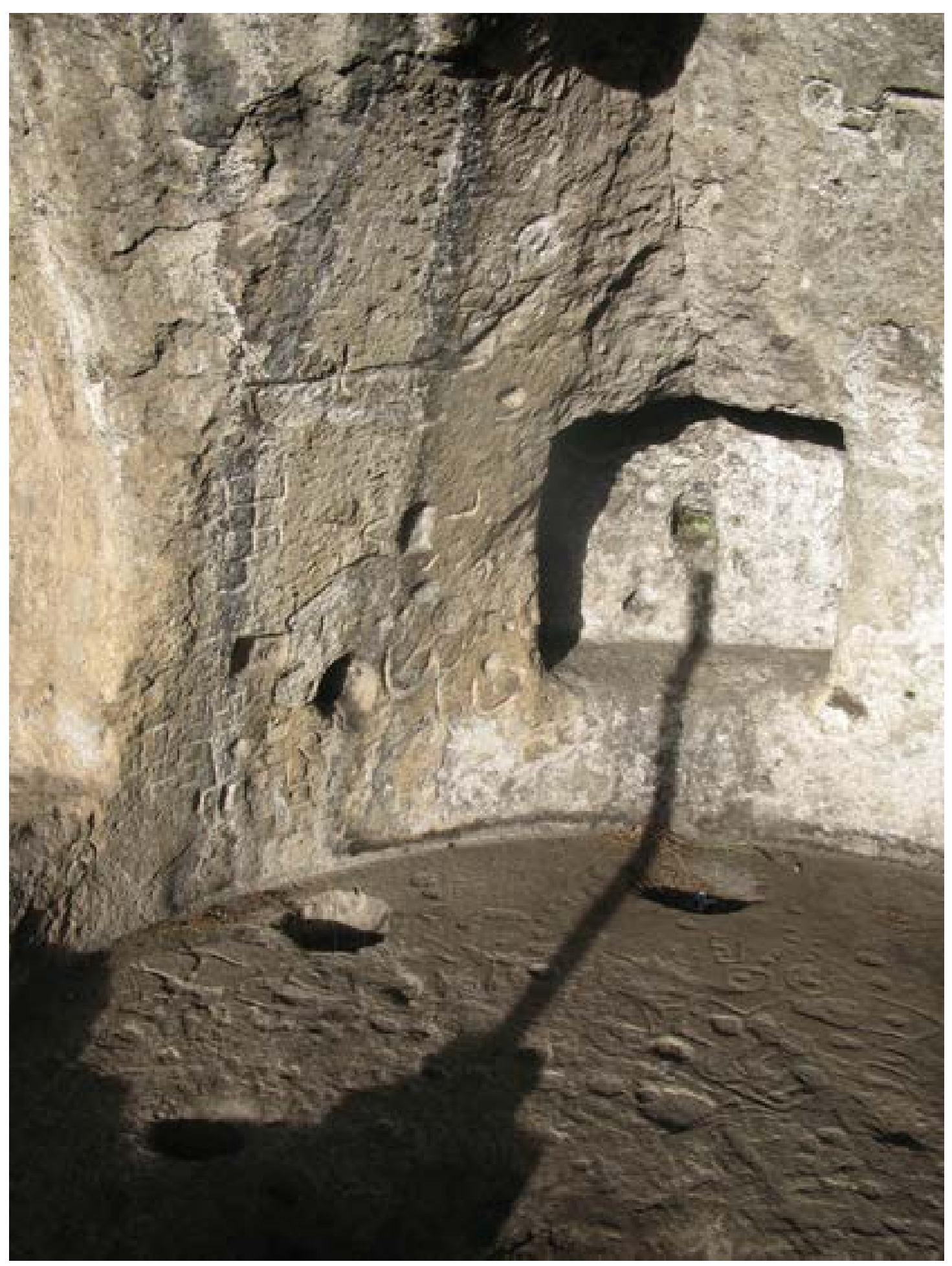

Figura $\mathrm{N}^{\circ} 4$. La segunda cueva vía al canal, nótese la simbología vaginal

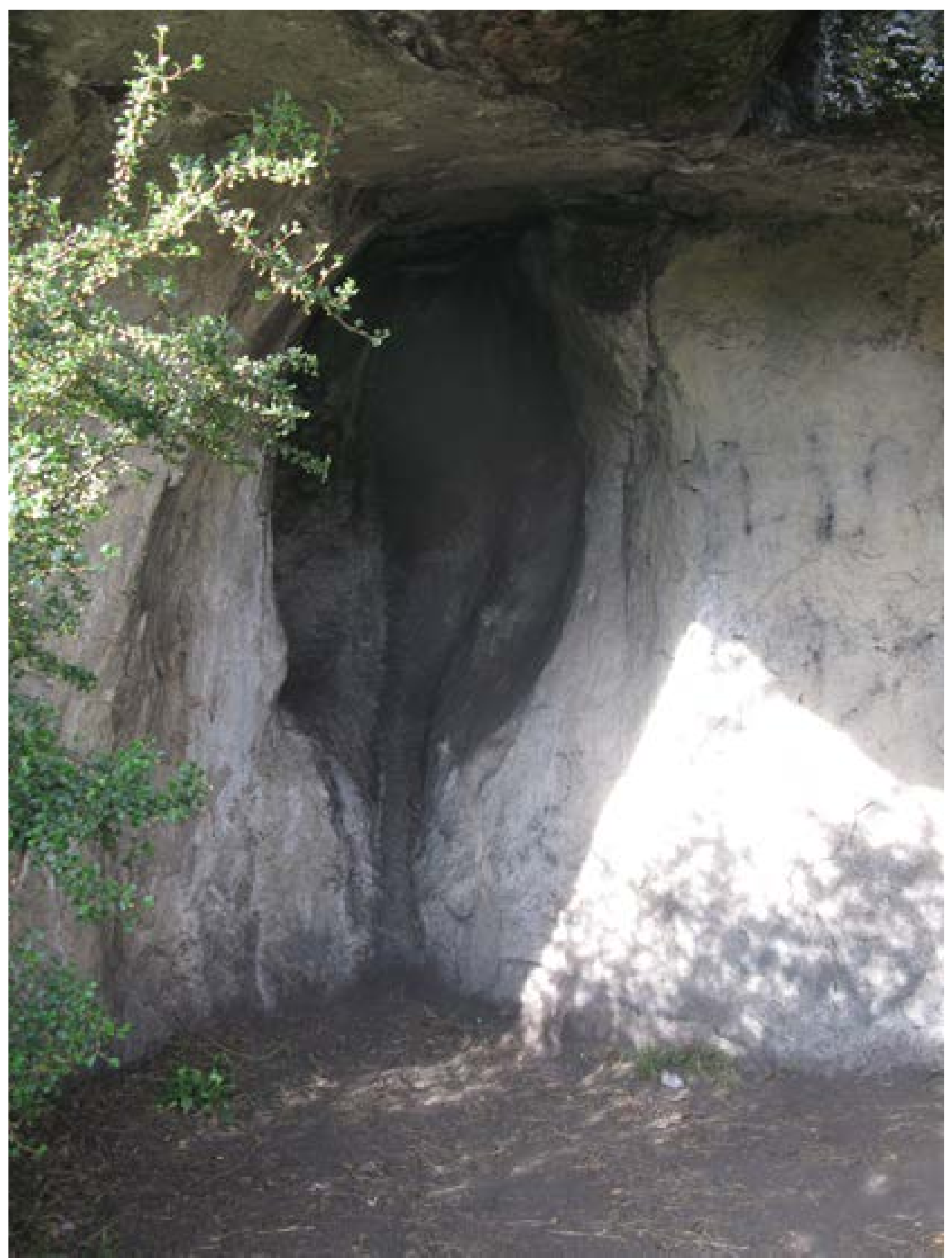


Figura $\mathrm{N}^{\circ}$ 5. Petroglifos al ingreso de la segunda cueva

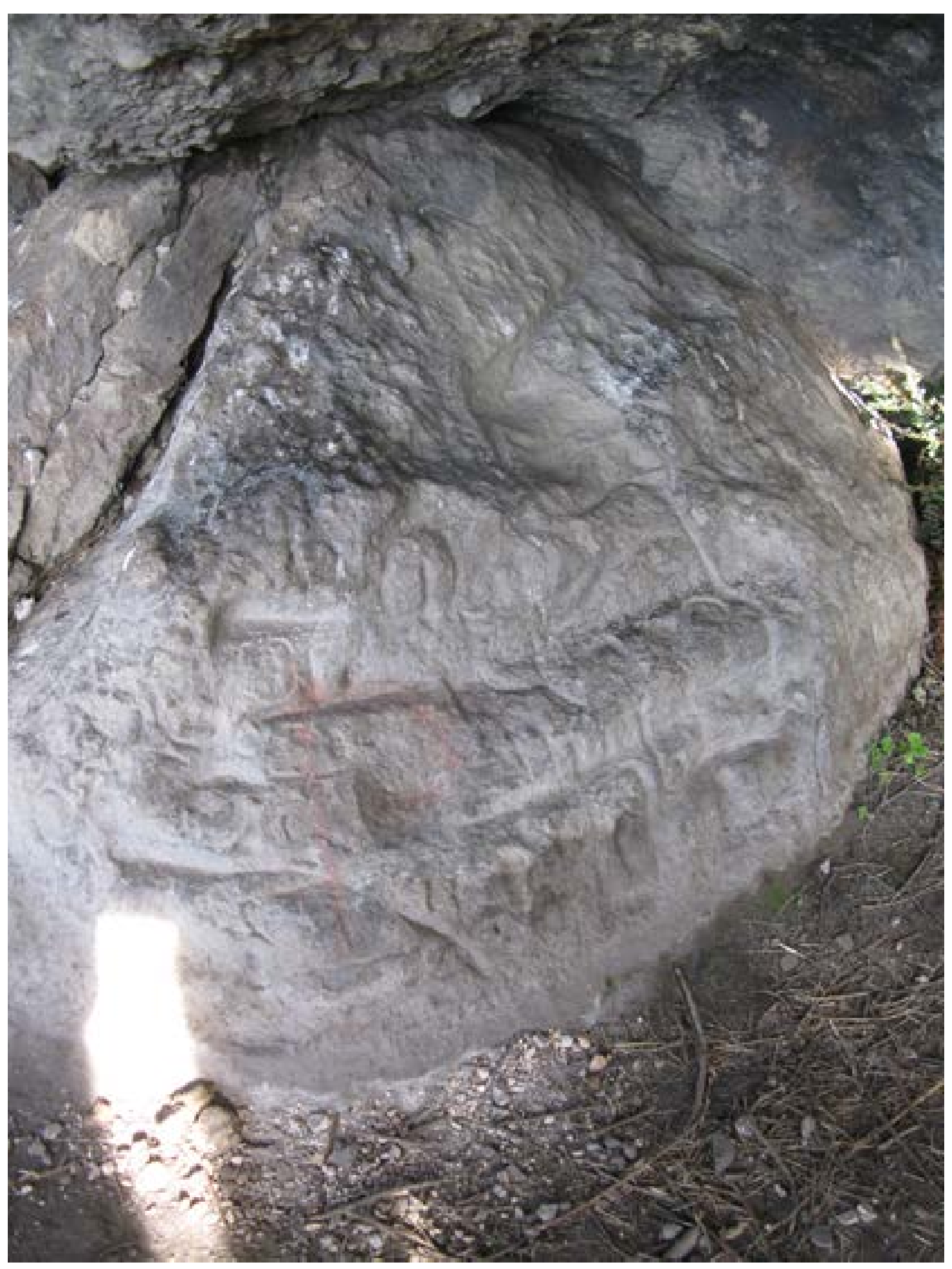

Figura $\mathrm{N}^{\circ}$ 6. Inicios del segmento I del Canal

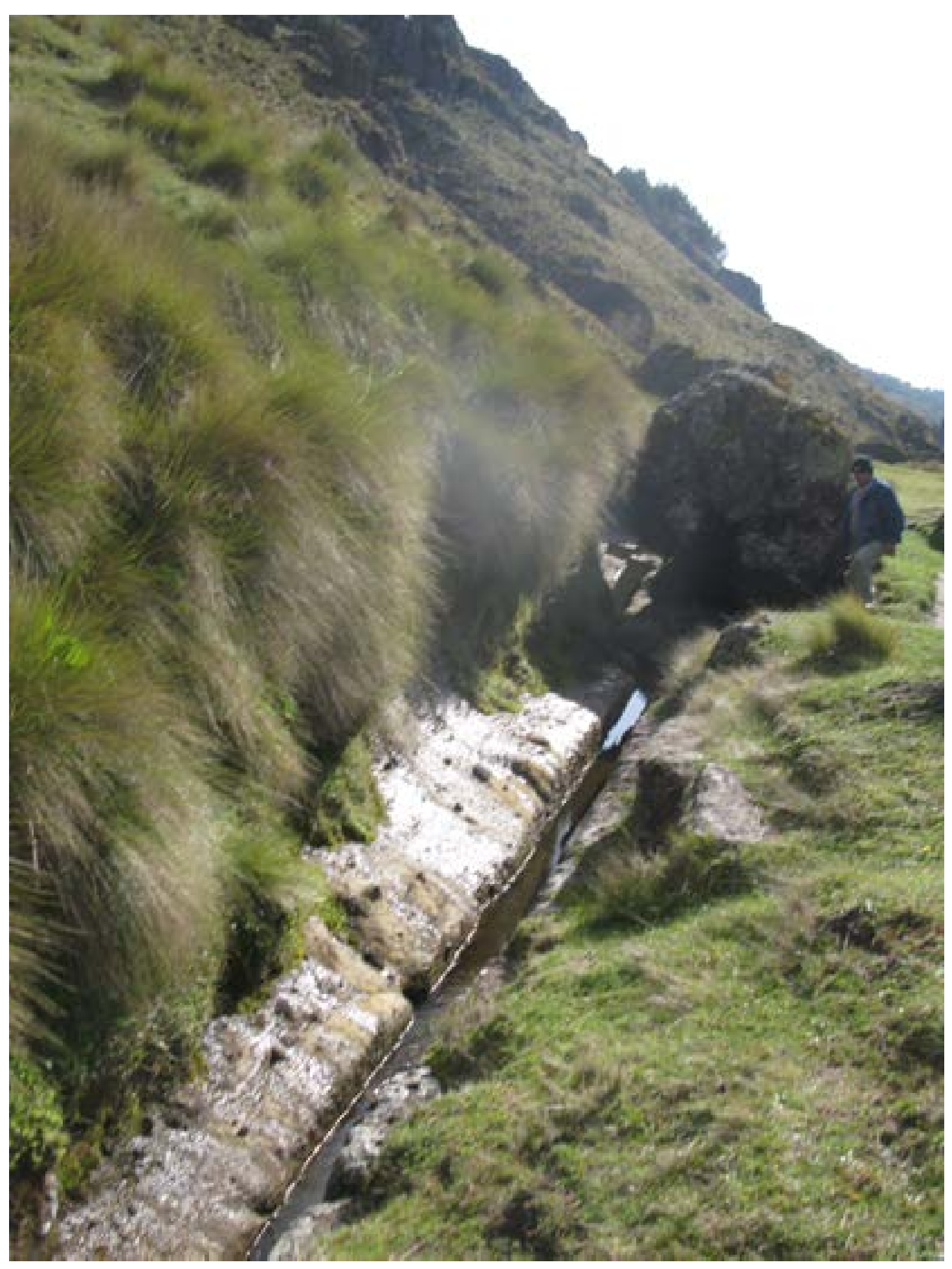


Figura $\mathrm{N}^{\circ} 7$. Inicios del segmento I del Canal

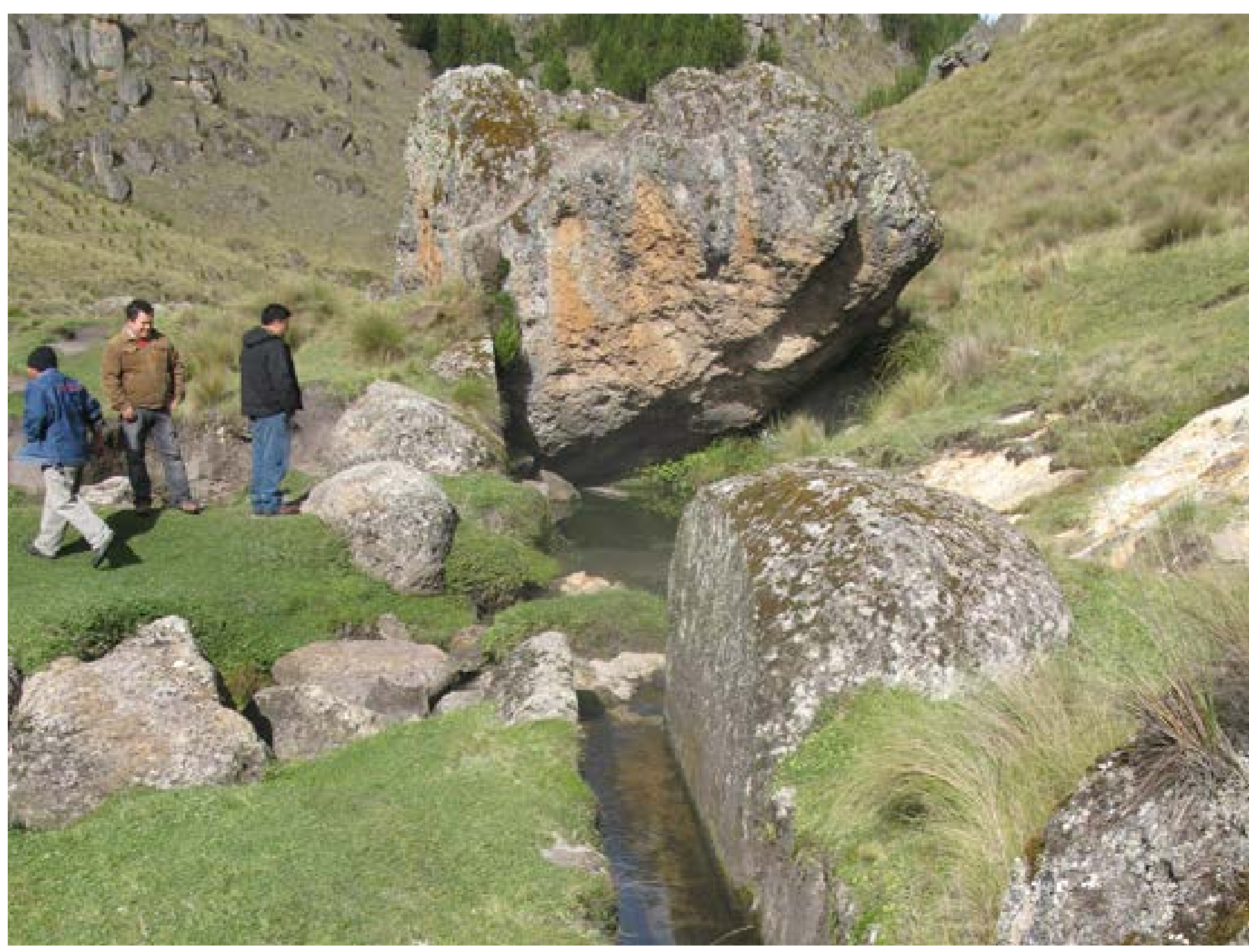

Figura $\mathrm{N}^{\circ}$ 8. Curvas y líneas rectas en el trazo del canal

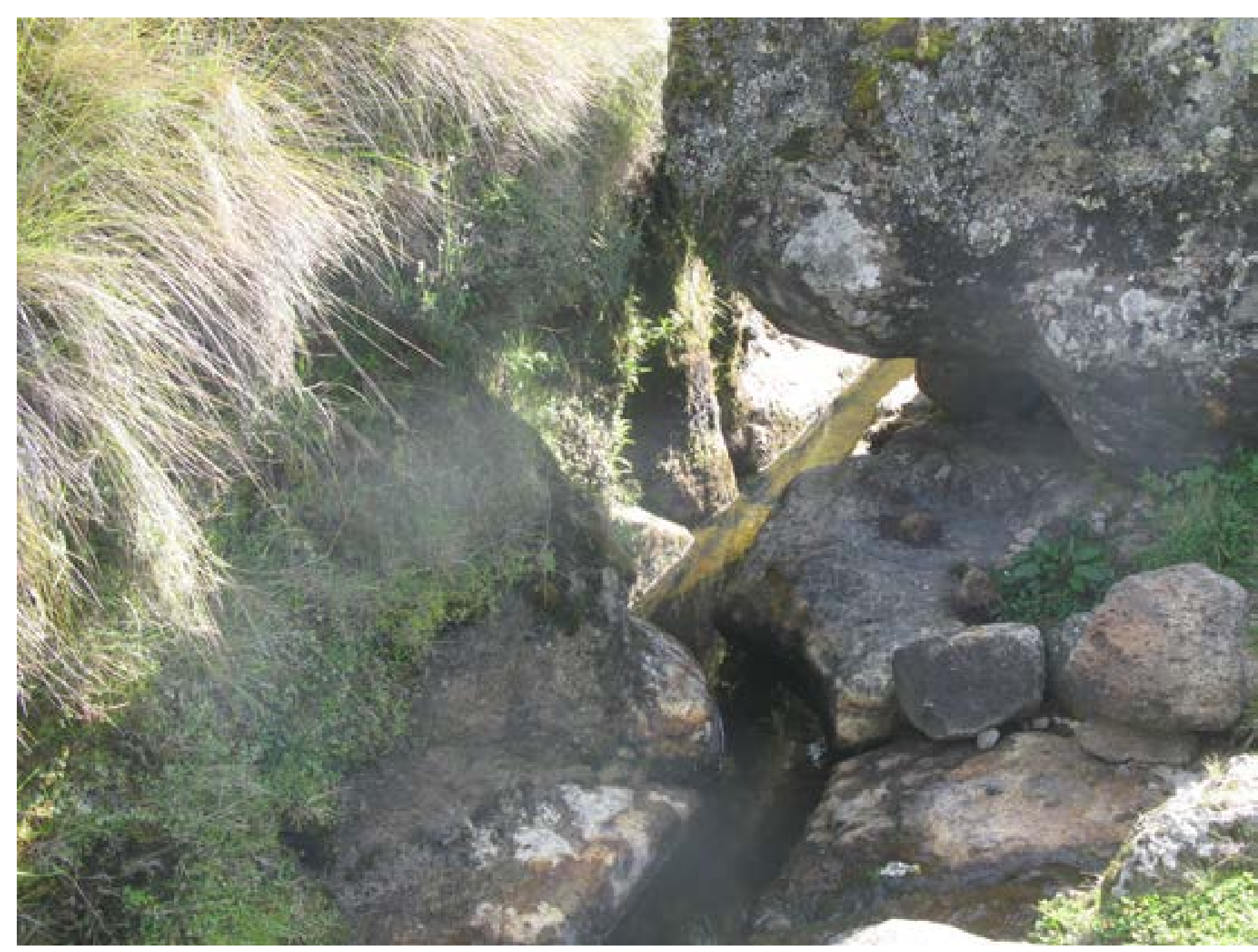


Figura $\mathrm{N}^{\circ}$ 9. Ángulos rectos, no necesarios para disminuir la velocidad del caudal.

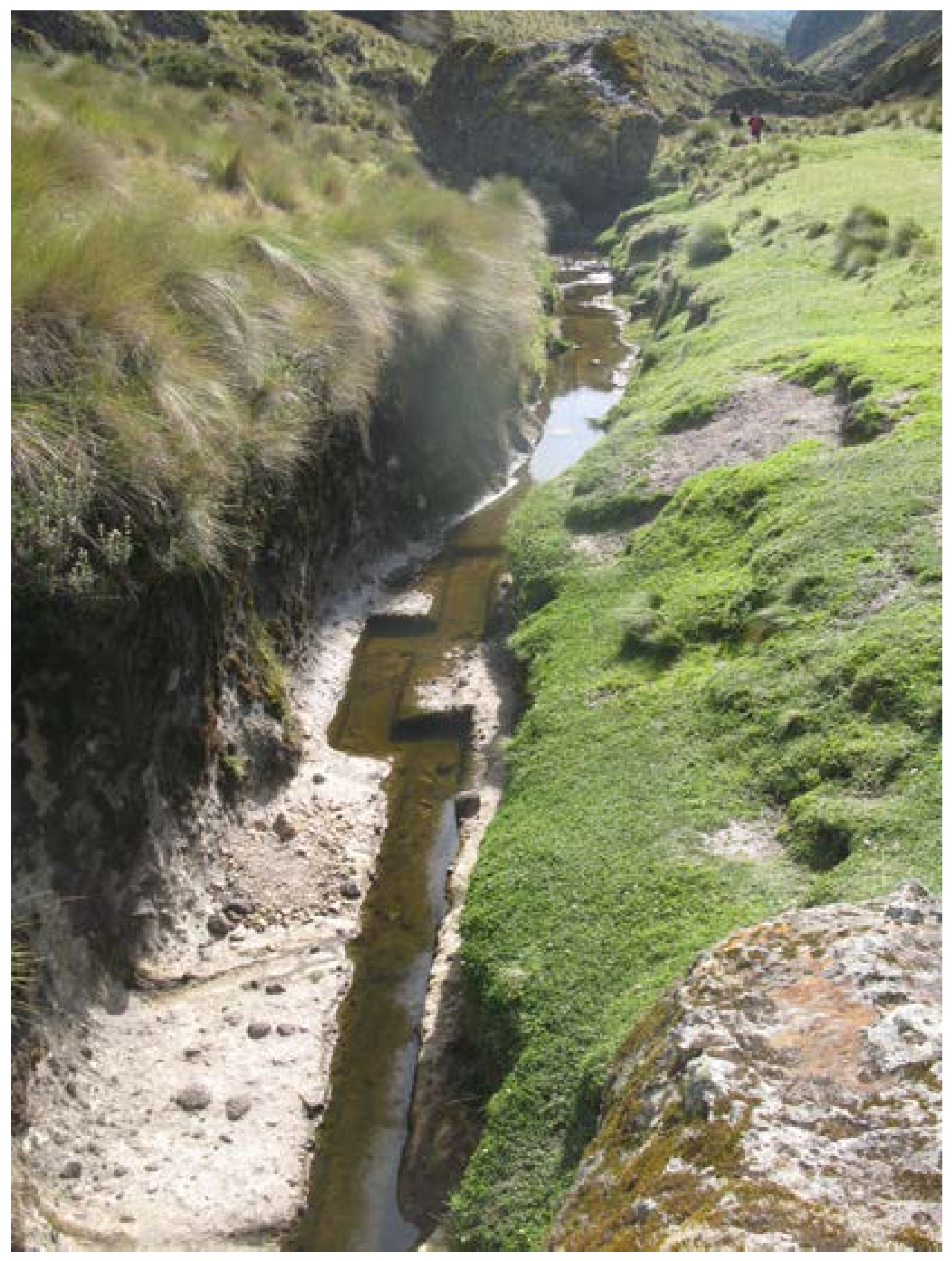

Figura $\mathrm{N}^{\circ} 10$. Aproximación de la vista anterior (9).

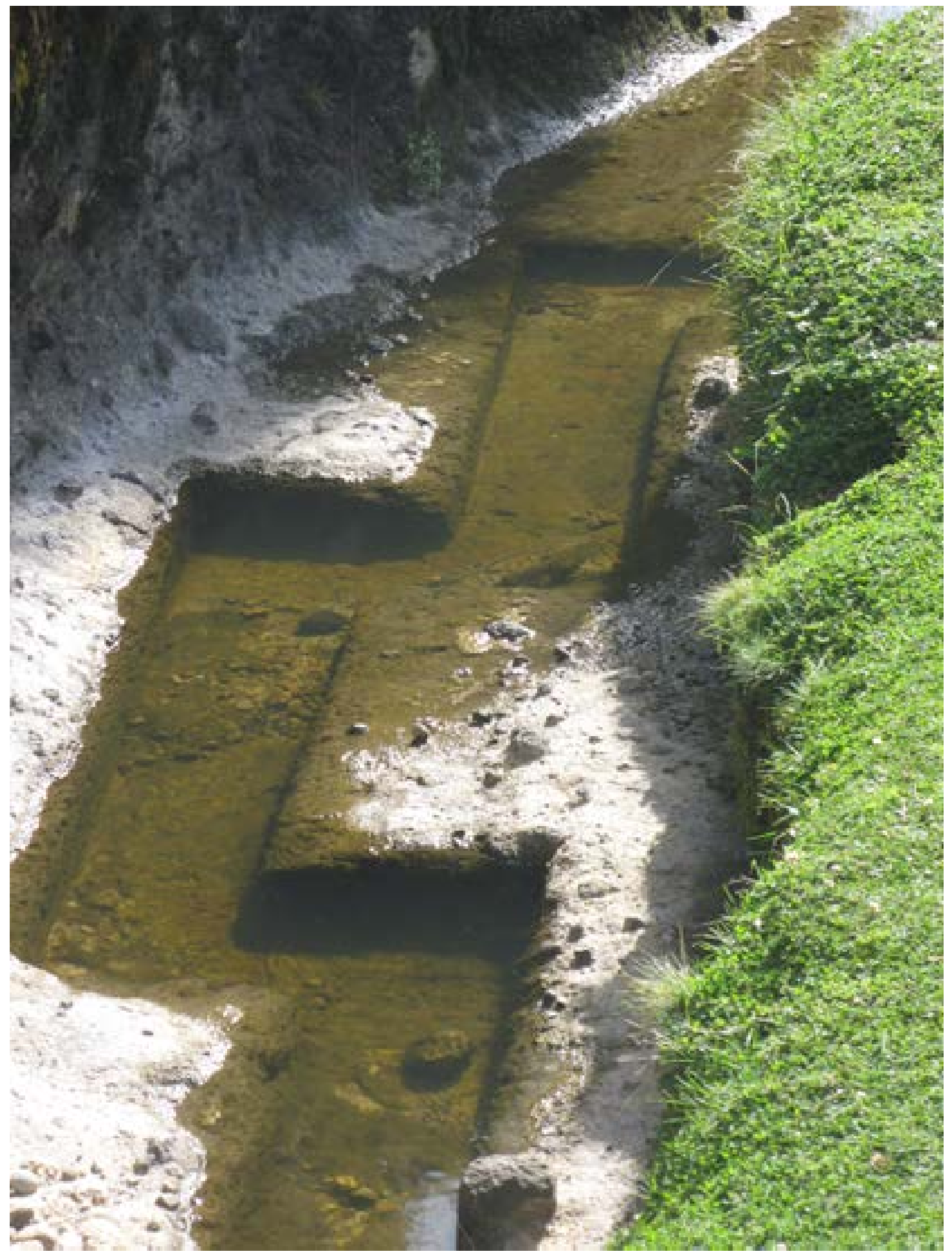


Figura $\mathrm{N}^{\circ} 11$. Trazos en la roca del Segmento I del Canal.

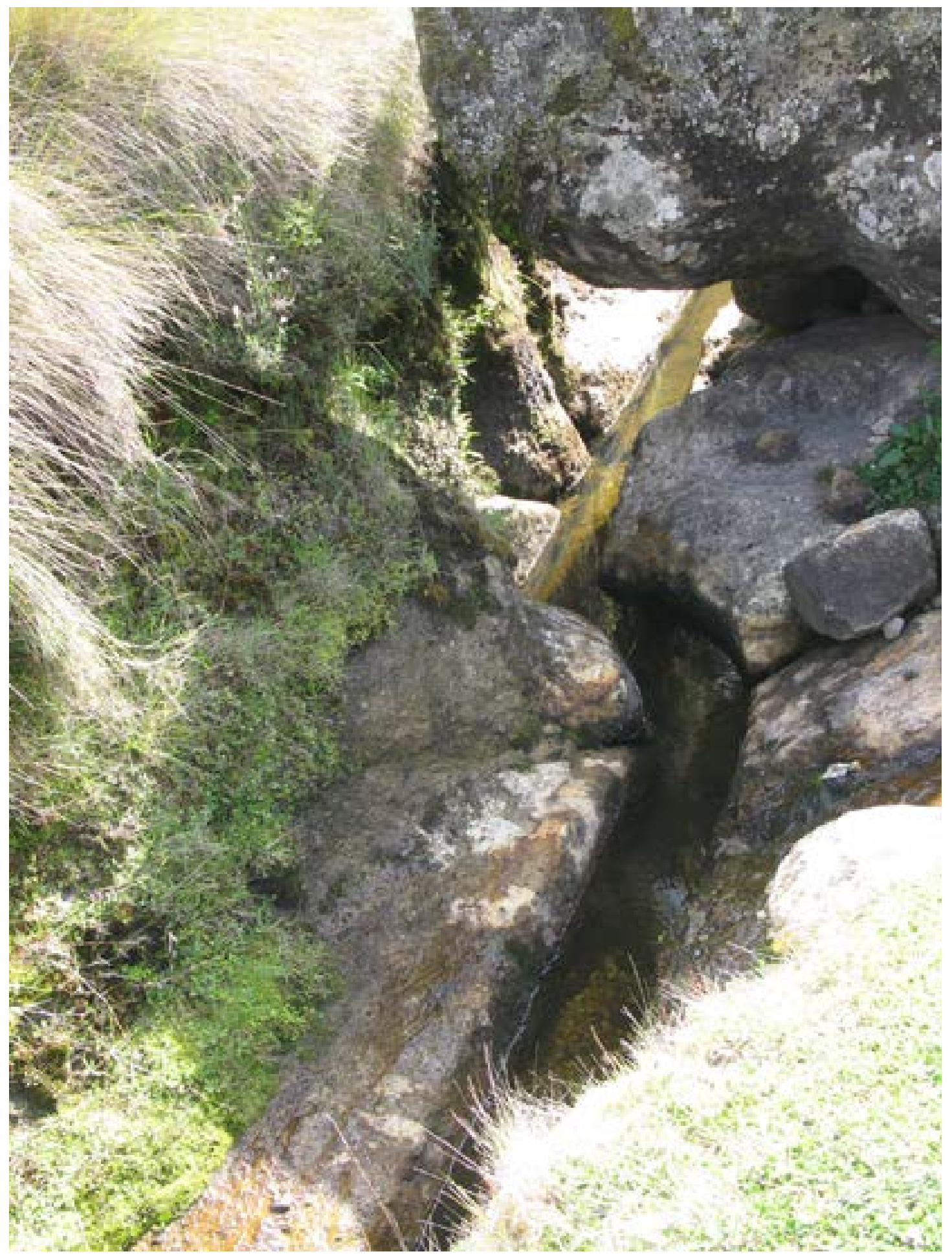

Figura $\mathrm{N}^{\circ}$ 12. Trazos en la roca del Segmento I del Canal.

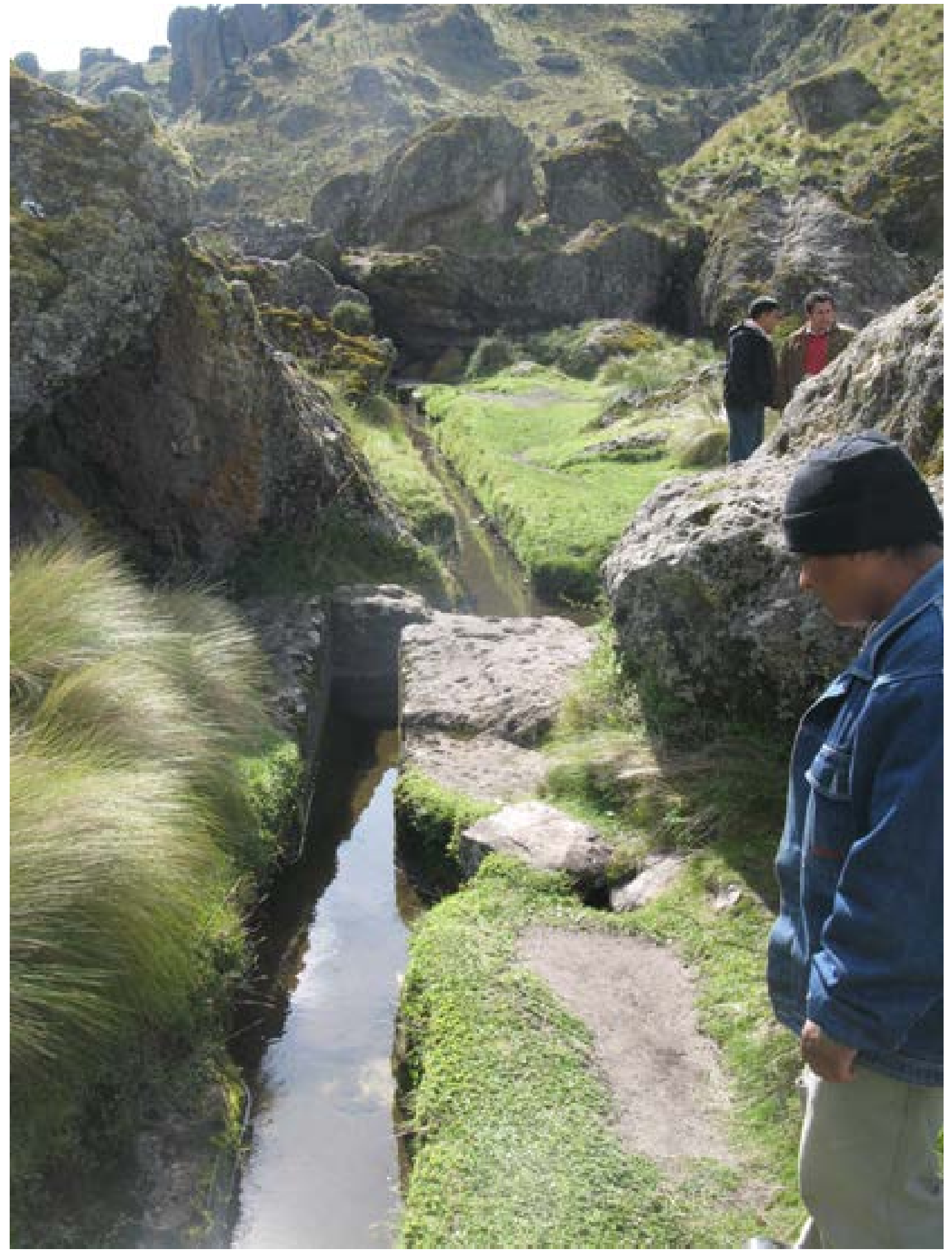


Figura $\mathrm{N}^{\circ} 13$. Roca tallada para la continuidad del canal manteniendo la pendiente de $0.001,5 \mathrm{~mm}$

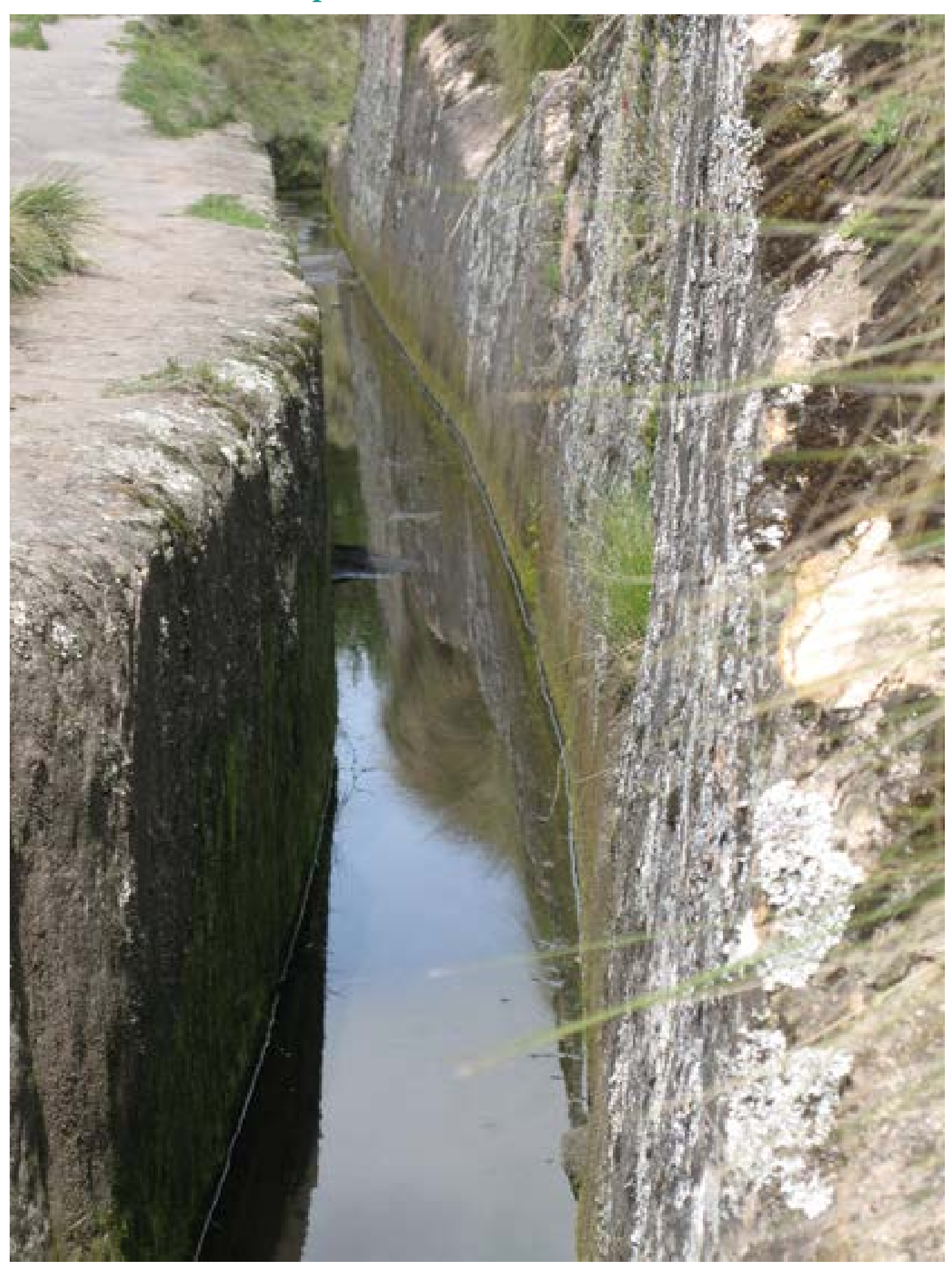

Figura $\mathrm{N}^{\circ}$ 14. Línea recta, de culminación del segmento I del canal.

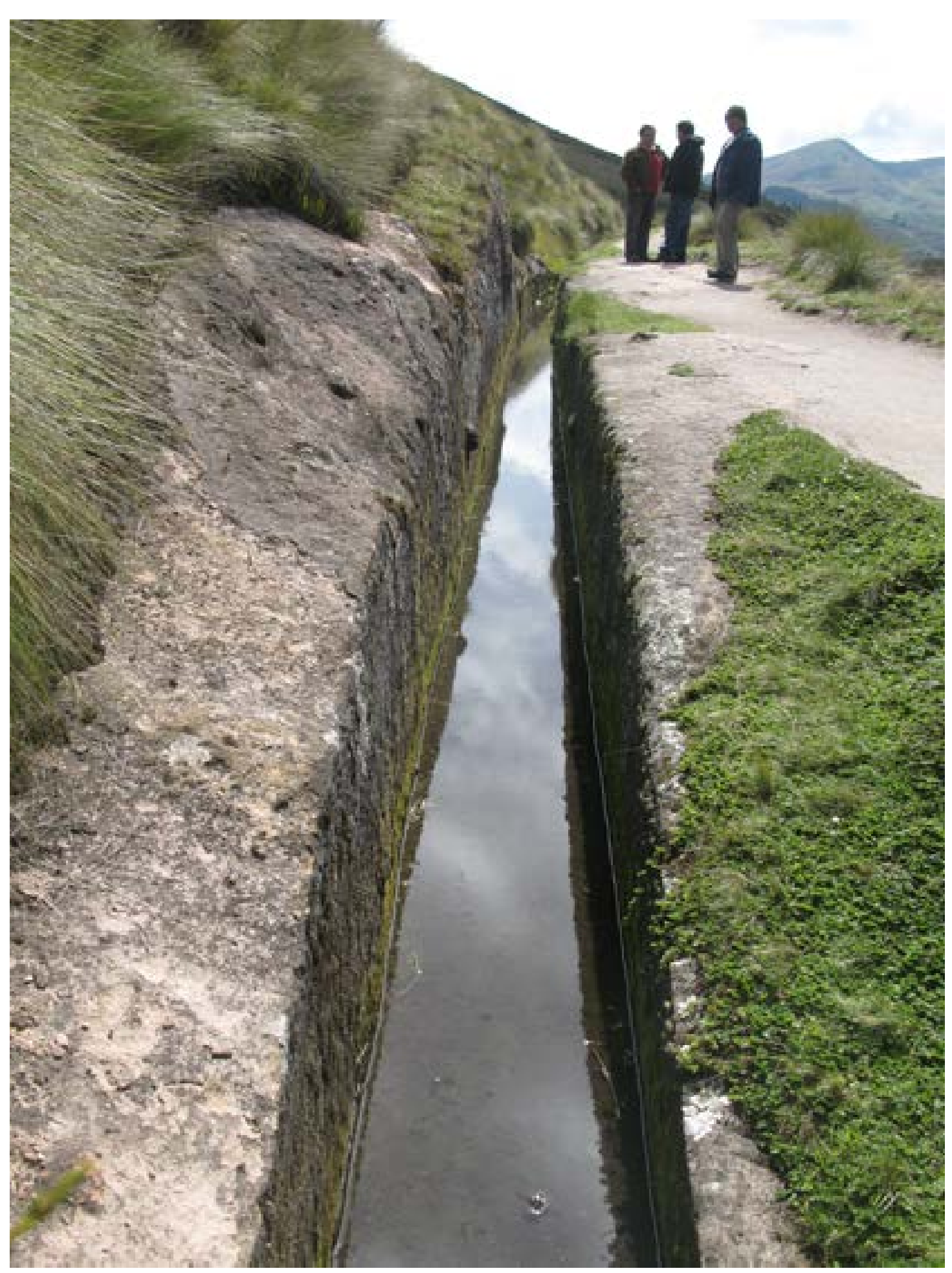


Figura $\mathrm{N}^{\circ}$ 16. Inicio del segmento II del canal o proyección. Conserva similares características en su trazo y los petroglifos son más definidos, como la cruz andina.

Figura $\mathrm{N}^{\circ} 15$. Bloque solitario con diversidad de petroglifos, destacan dos pares de sandalias, al parecer de mujer por sus proporciones (de objetivo desconocido).

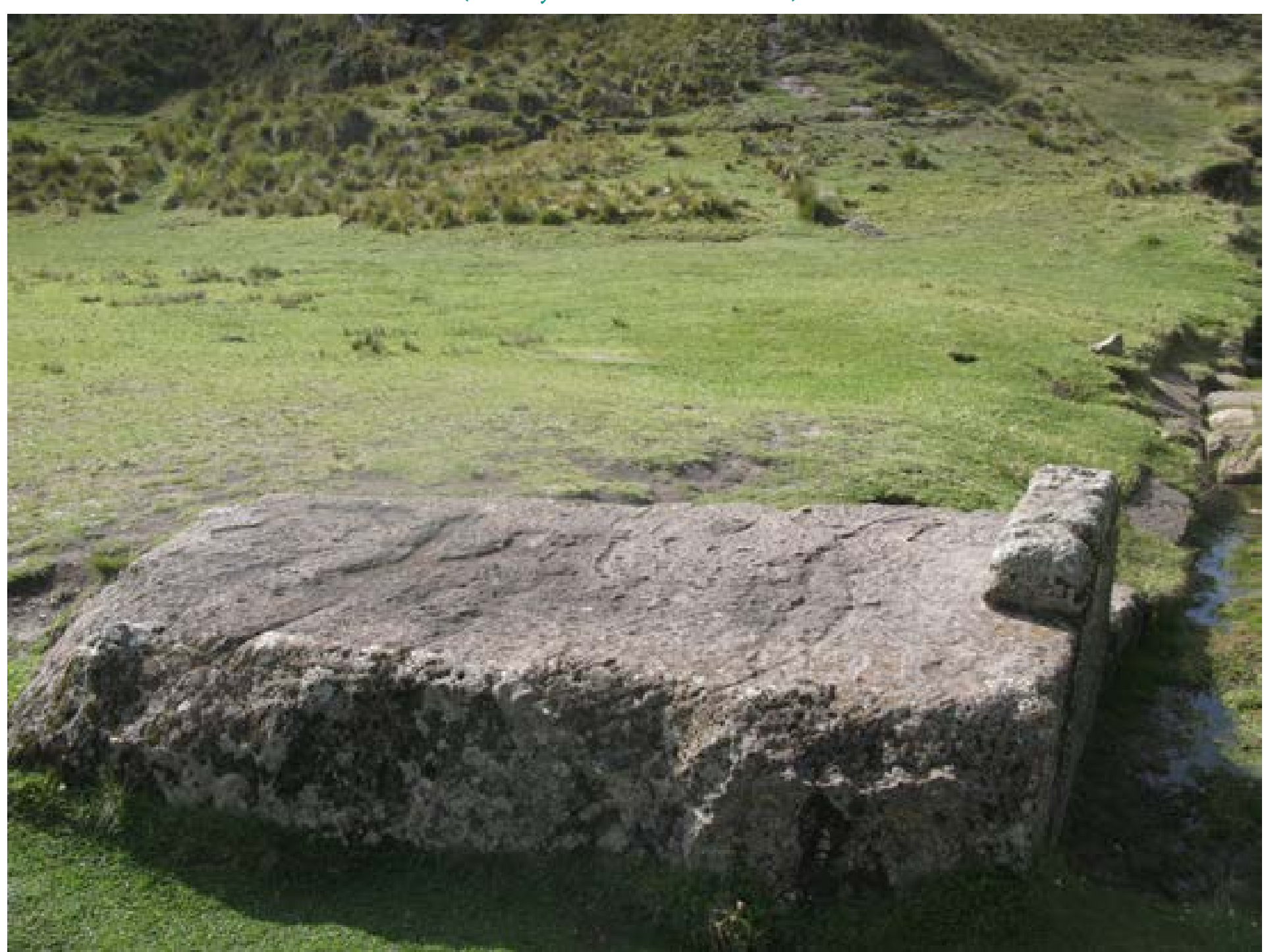

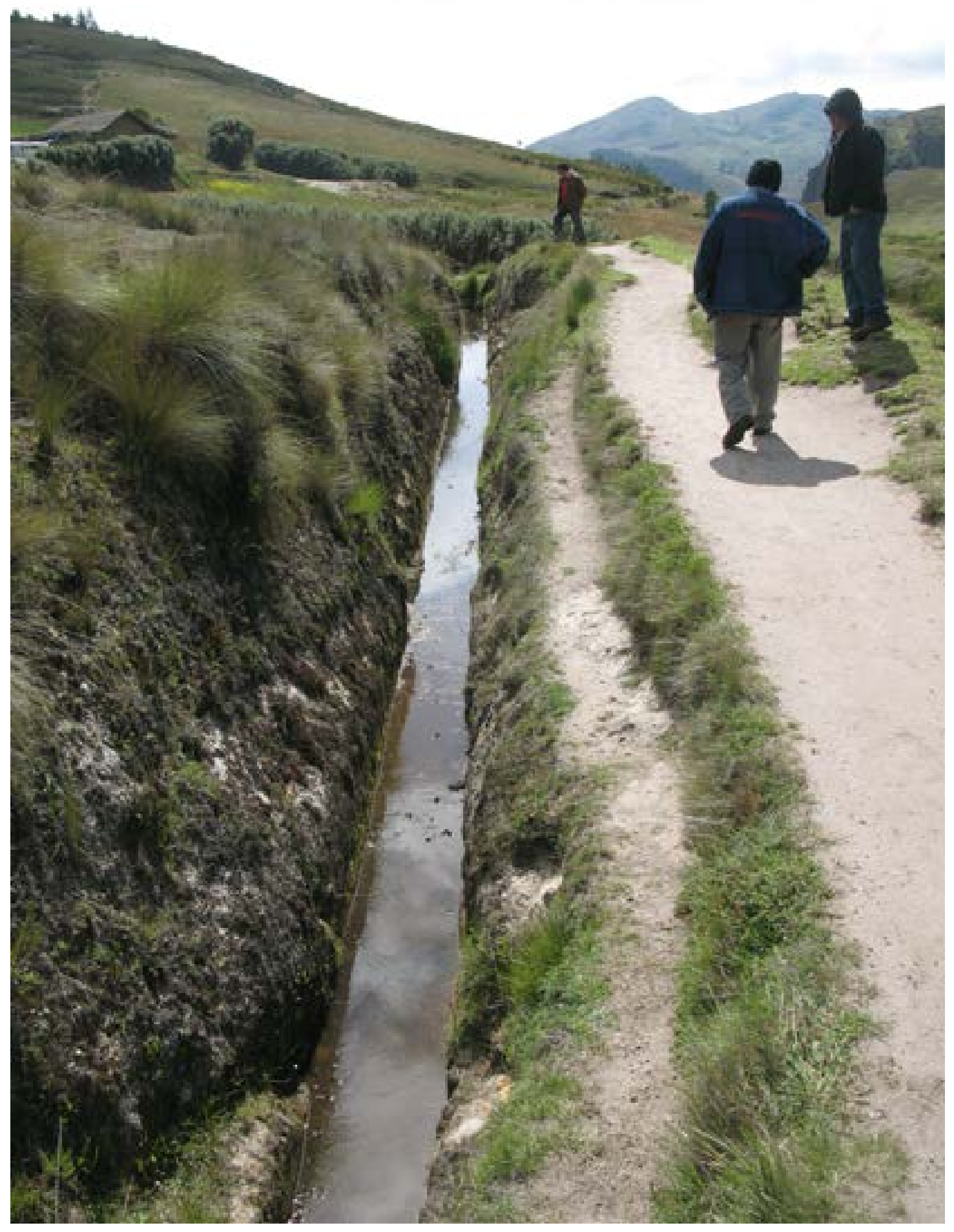


Figura $\mathrm{N}^{\circ}$ 17. El Segmento II del canal continúa sobre bases artificiales (rellenos) y excavación en la roca.

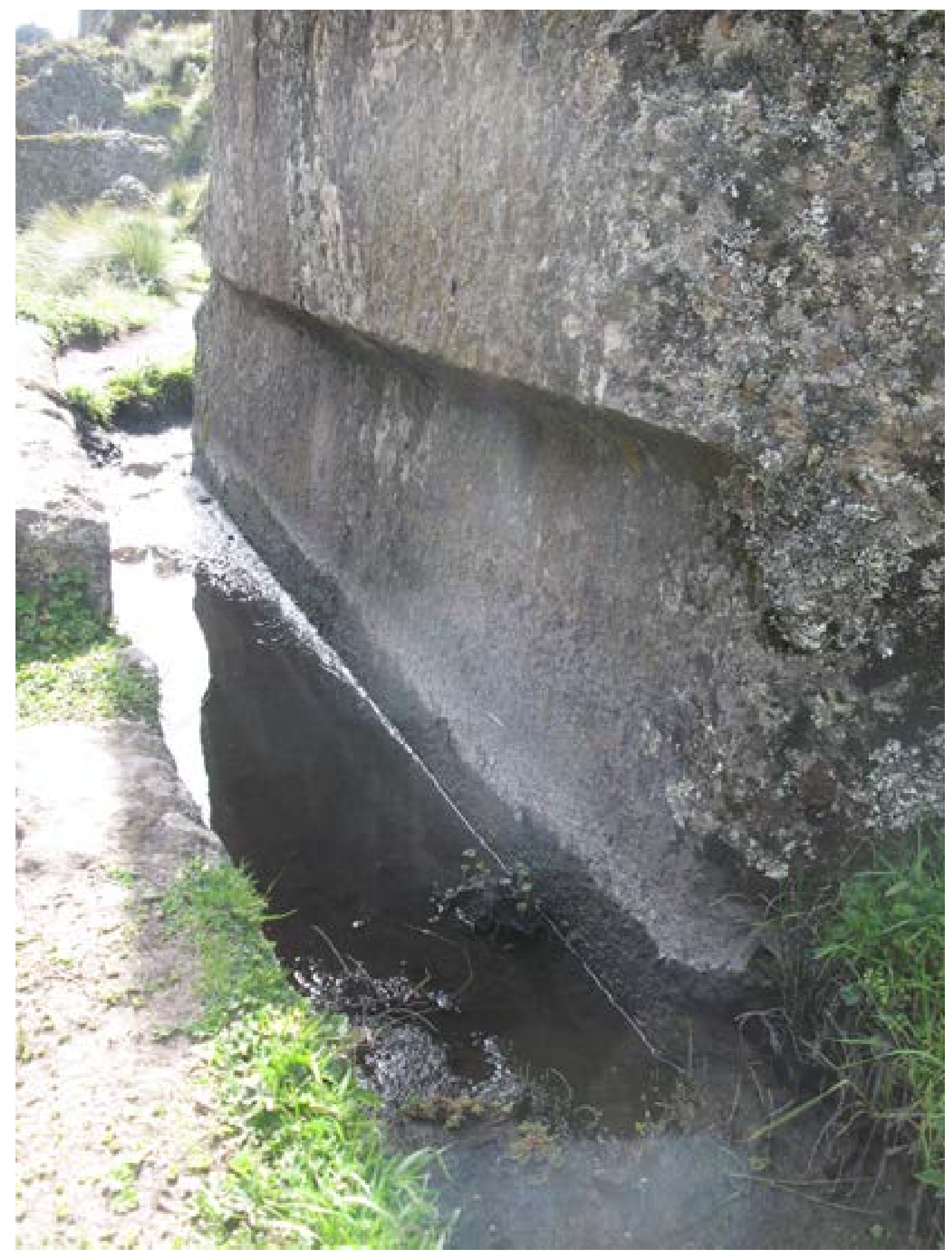

Figura $\mathrm{N}^{\circ}$ 18. Acueducto e ingreso de boca cuadrada, al túnel que atraviesa diez metros de la falda del cerro.

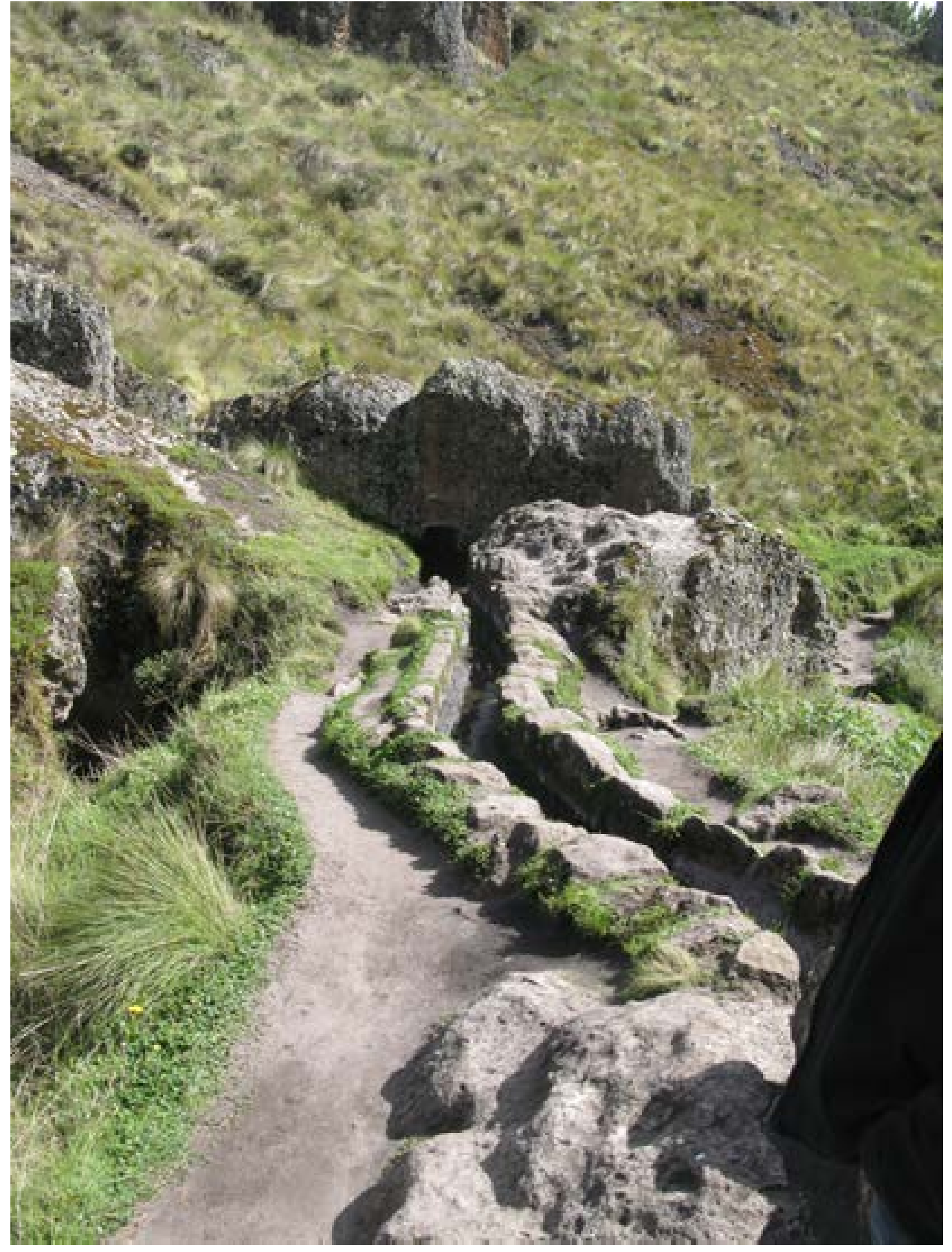


Figura $\mathrm{N}^{\circ}$ 19. Salida del Boca rectangular, del túnel. $(0.80 \times 0.60 \mathrm{~m}$. de alto)

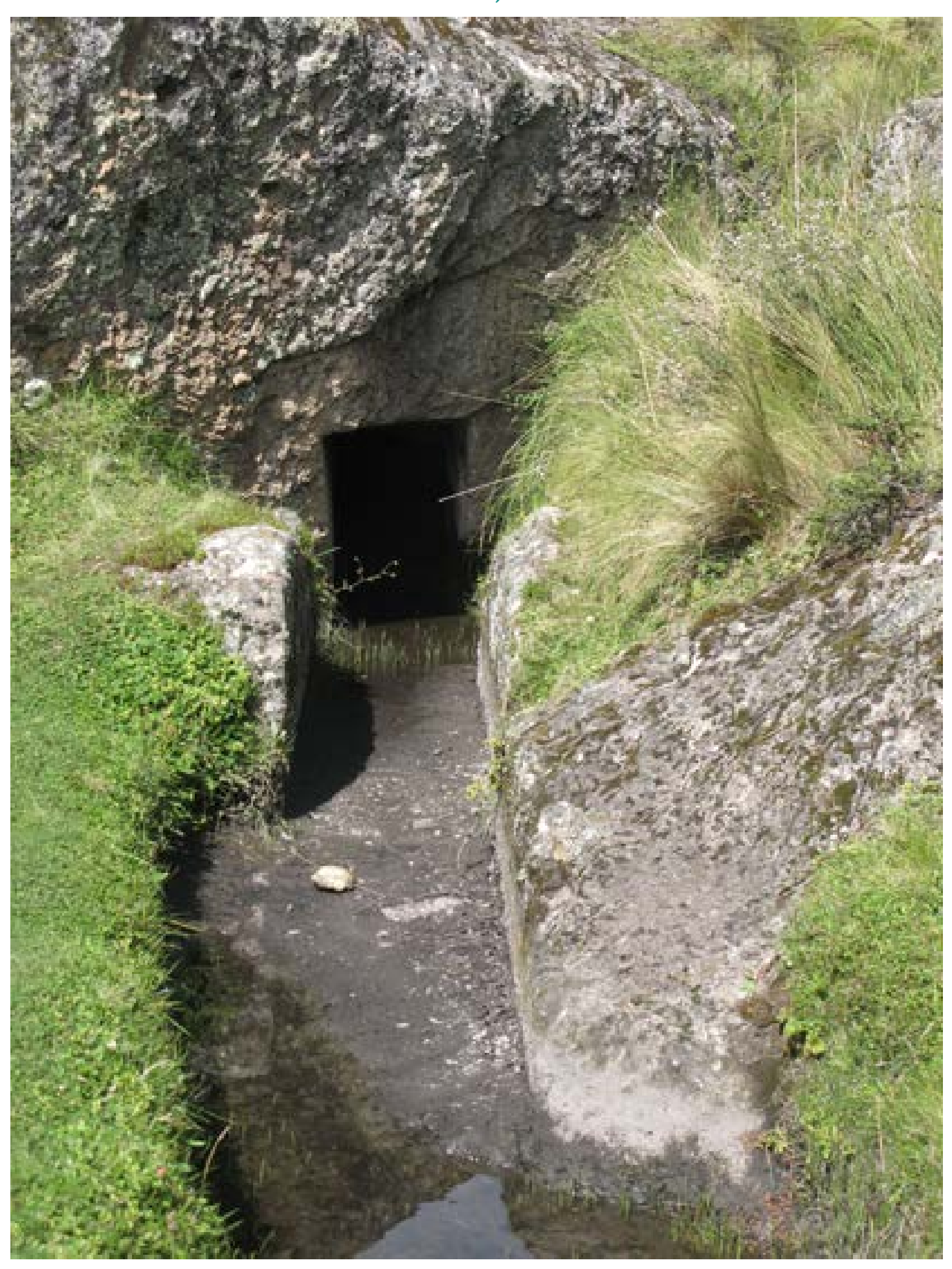

Figura $\mathrm{N}^{\circ} 20$. Corte en zigzag de la falda del cerro con mural de petroglifos.

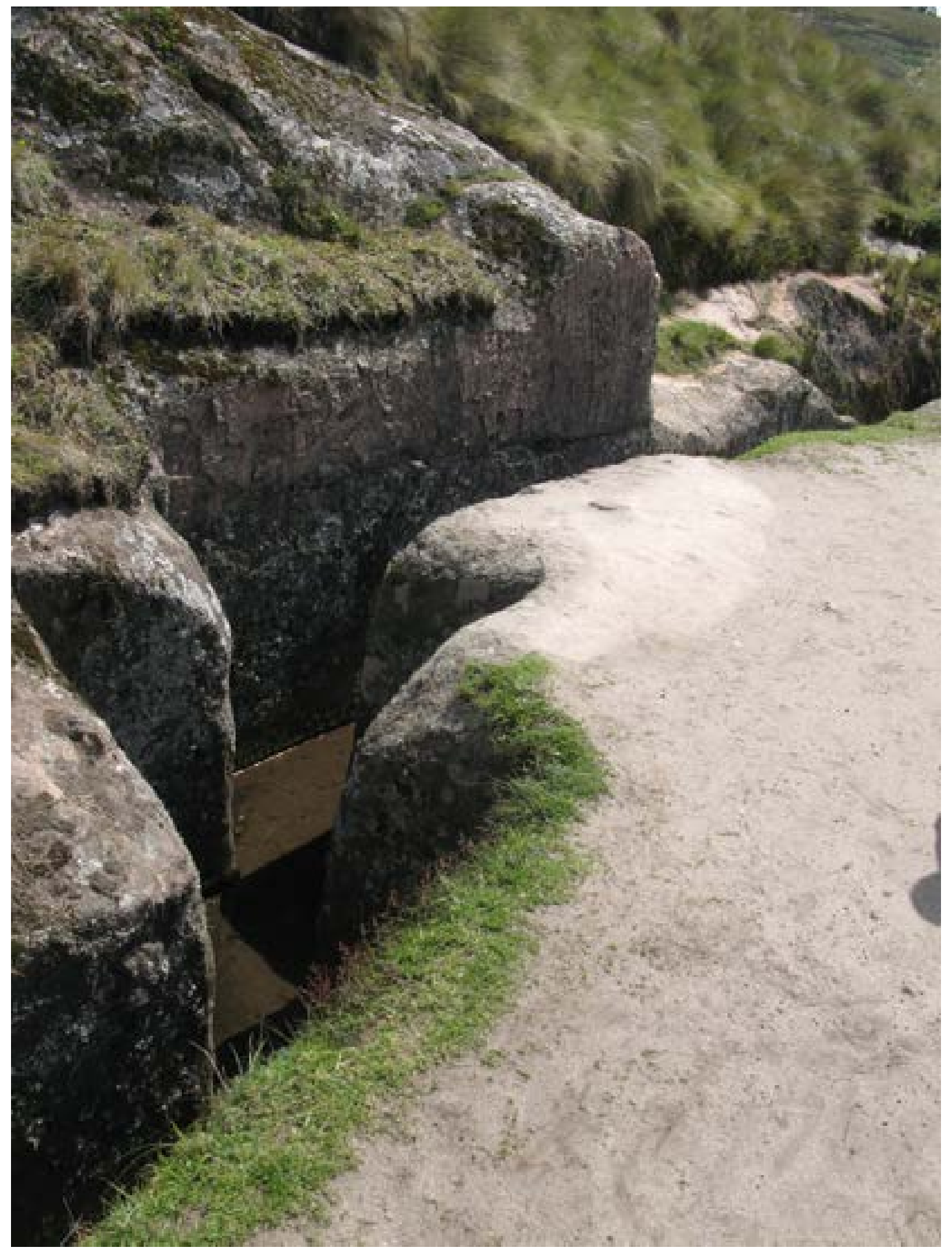


Figura $\mathrm{N}^{\circ}$ 21. Tercera cueva ubicada en el Segmento II del canal, en la base del frontis se observan petroglifos.

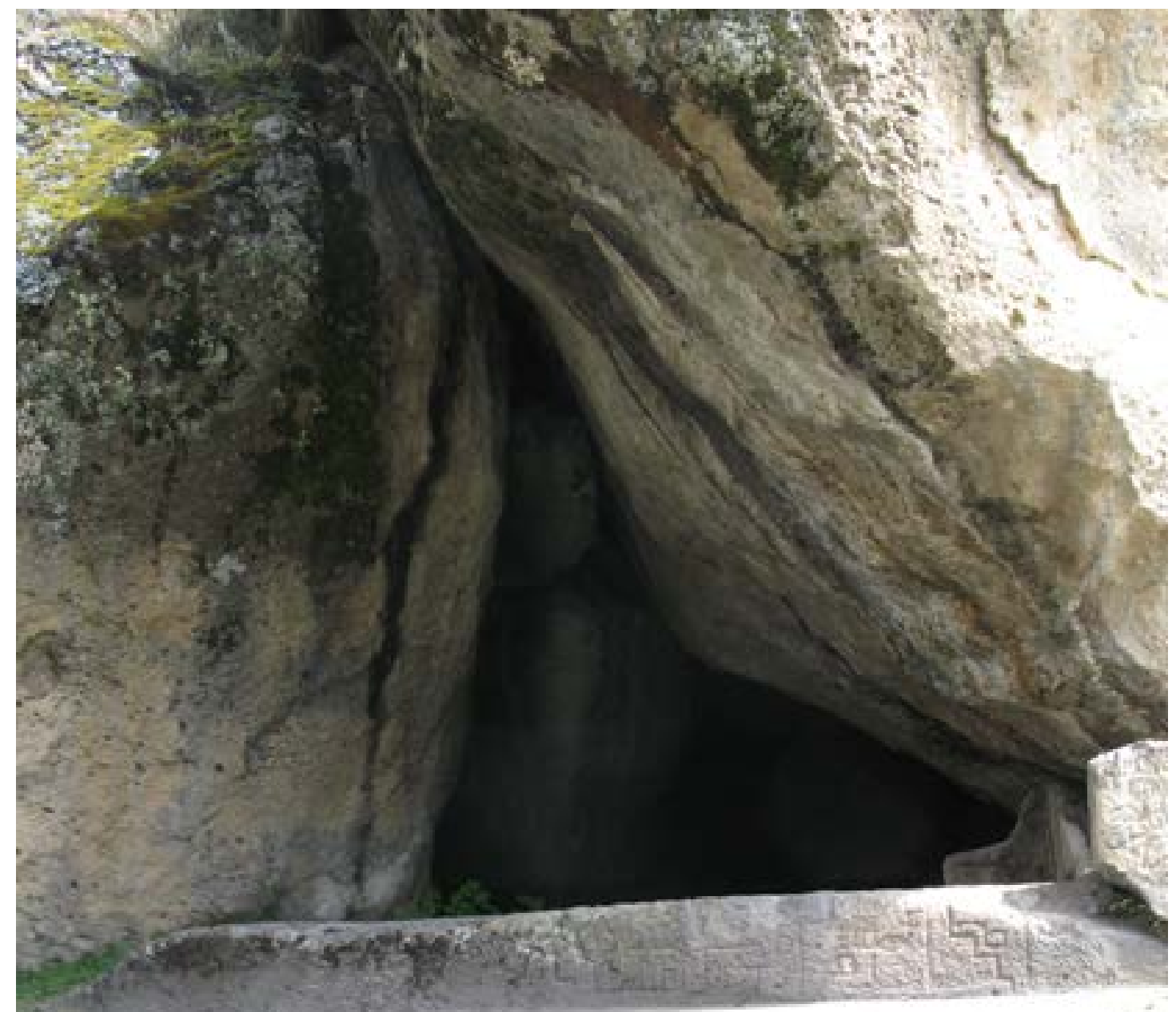

(4)

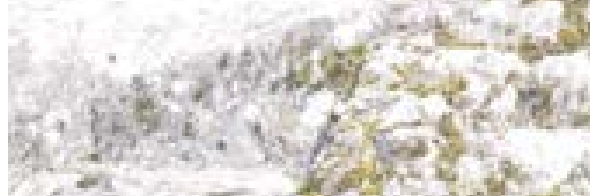

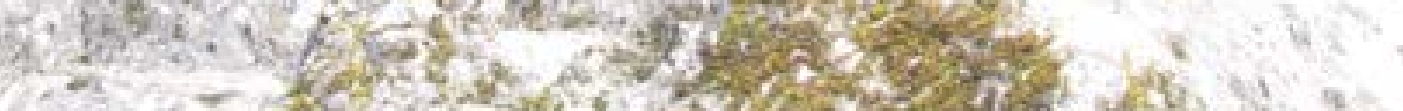

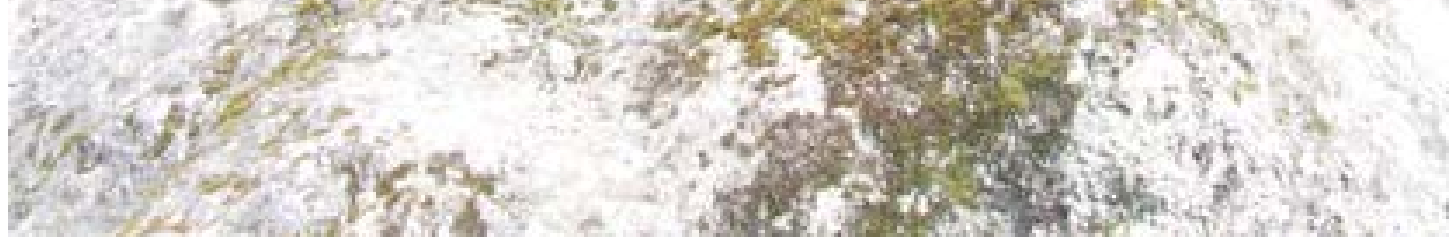

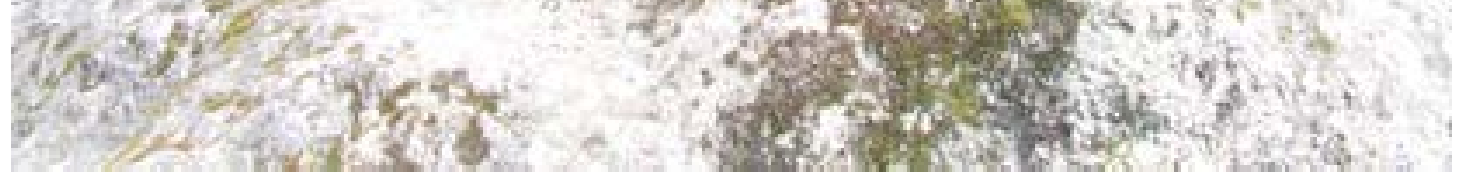

Figura $\mathrm{N}^{\circ} 22$. Piedra con figuras hieráticas al ingreso a la tercera cueva.

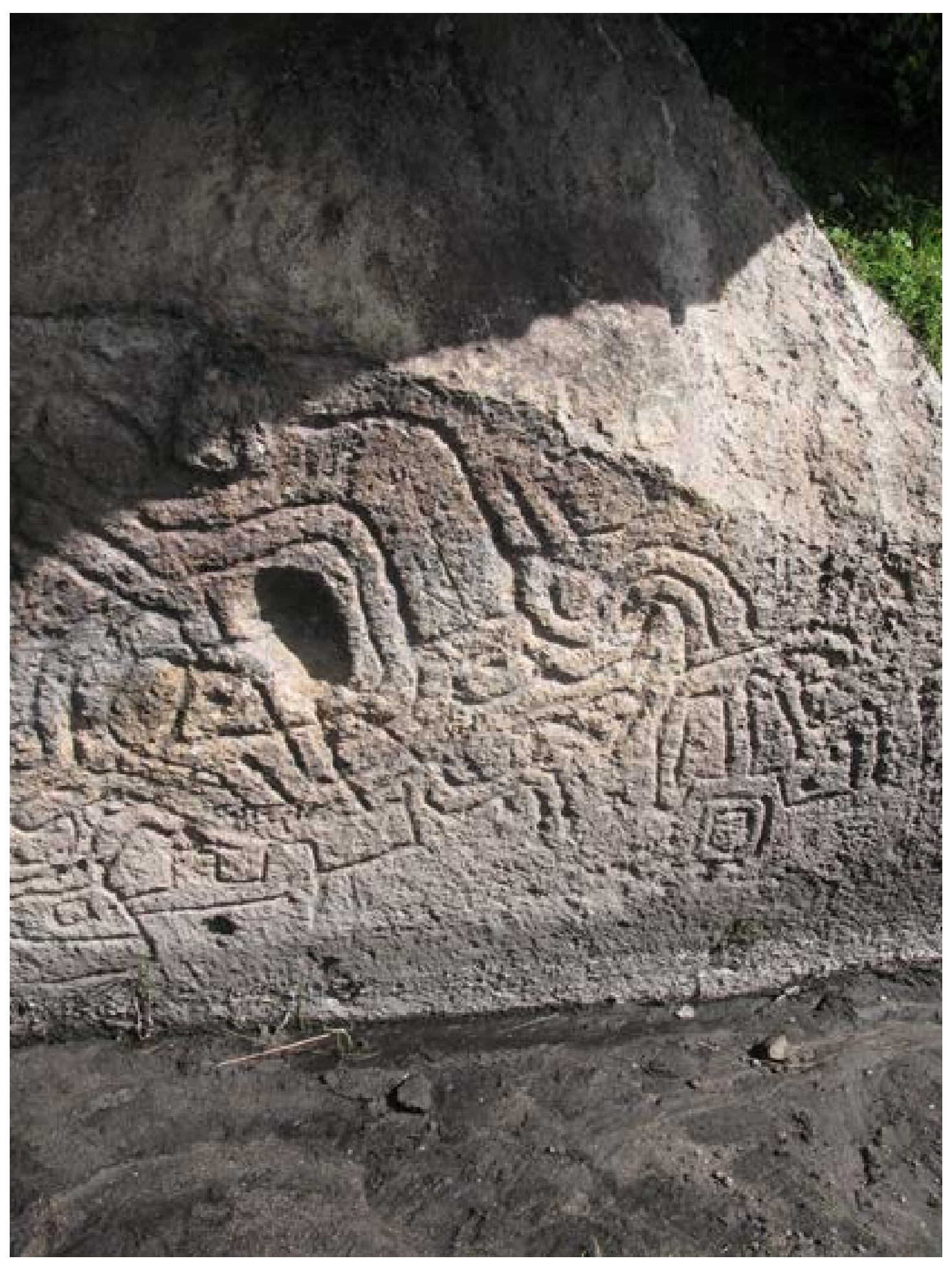


Figura $\mathrm{N}^{\circ} 23$. Figura de la cruz andina y Figura definida de concepto $(\dot{e}$ ?) en bajo relieve que acompaña al segmento II del canal.

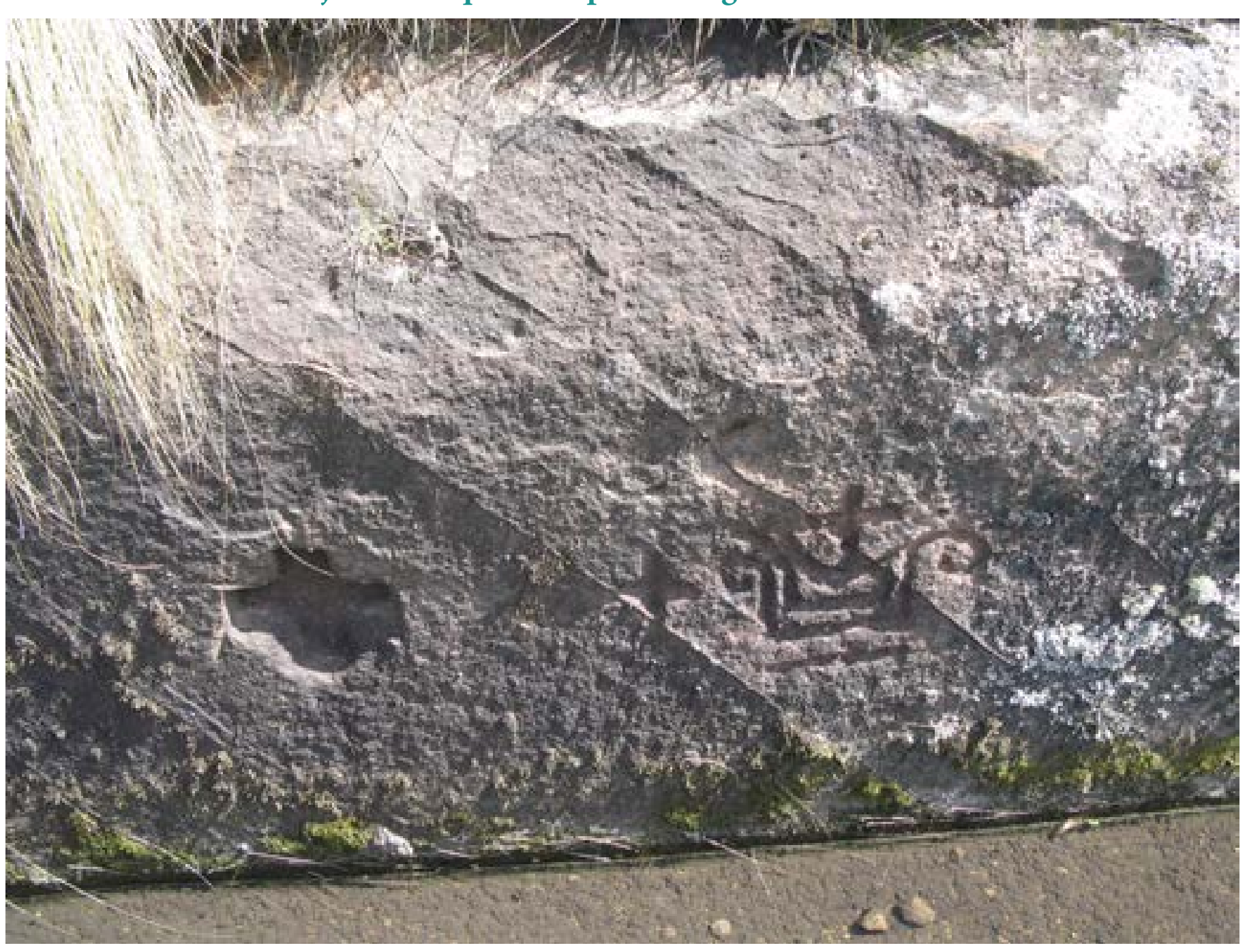

Figura $\mathrm{N}^{\circ}$ 24. Figura de la estrella rutilante, venus $\left(e^{e} ?\right)$

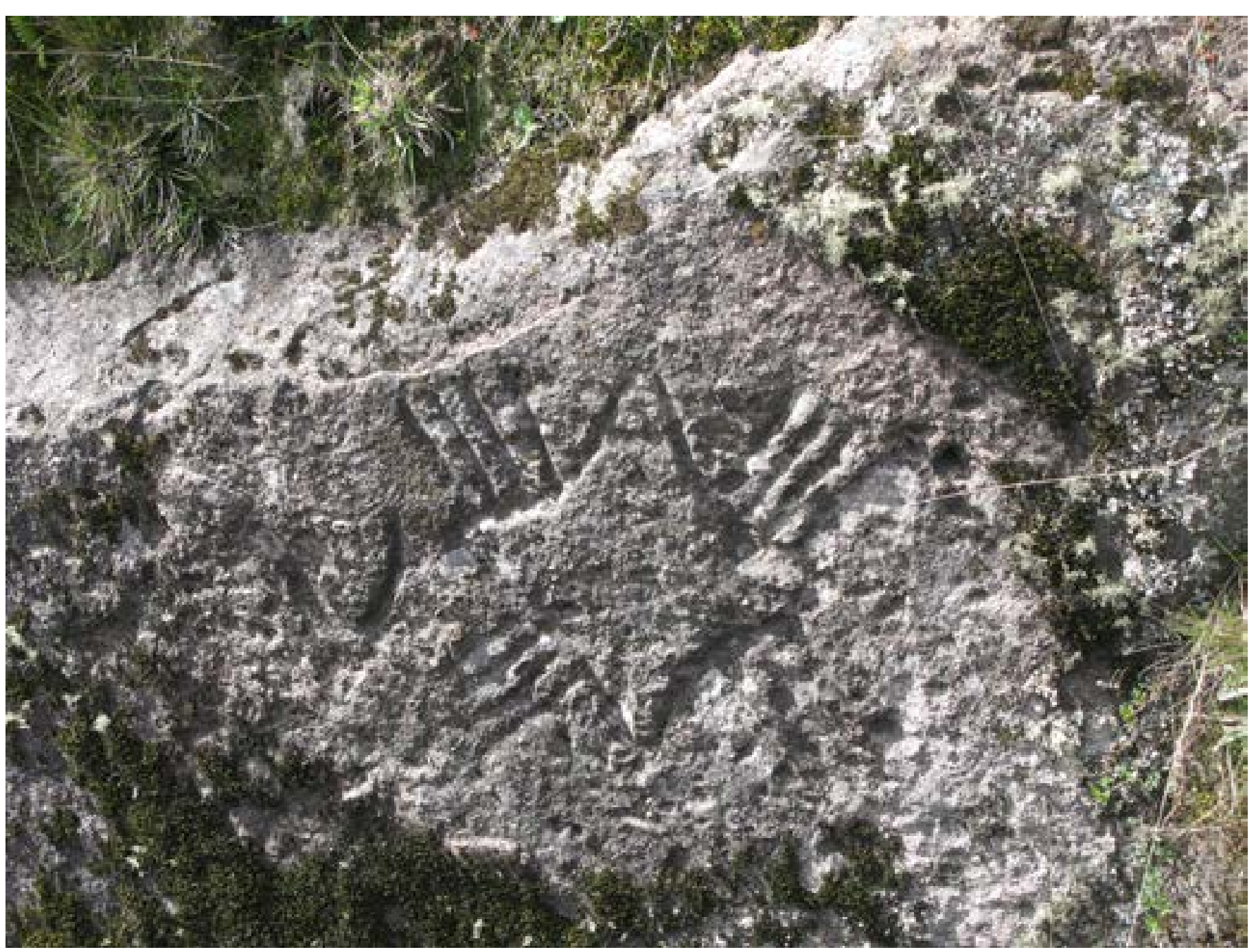


Figura $\mathrm{N}^{\circ} 26$. Segmento III del canal, hacia Layzón - Cajamarca, en esta-

Figura $\mathrm{N}^{\circ}$ 25. Las cruces andinas

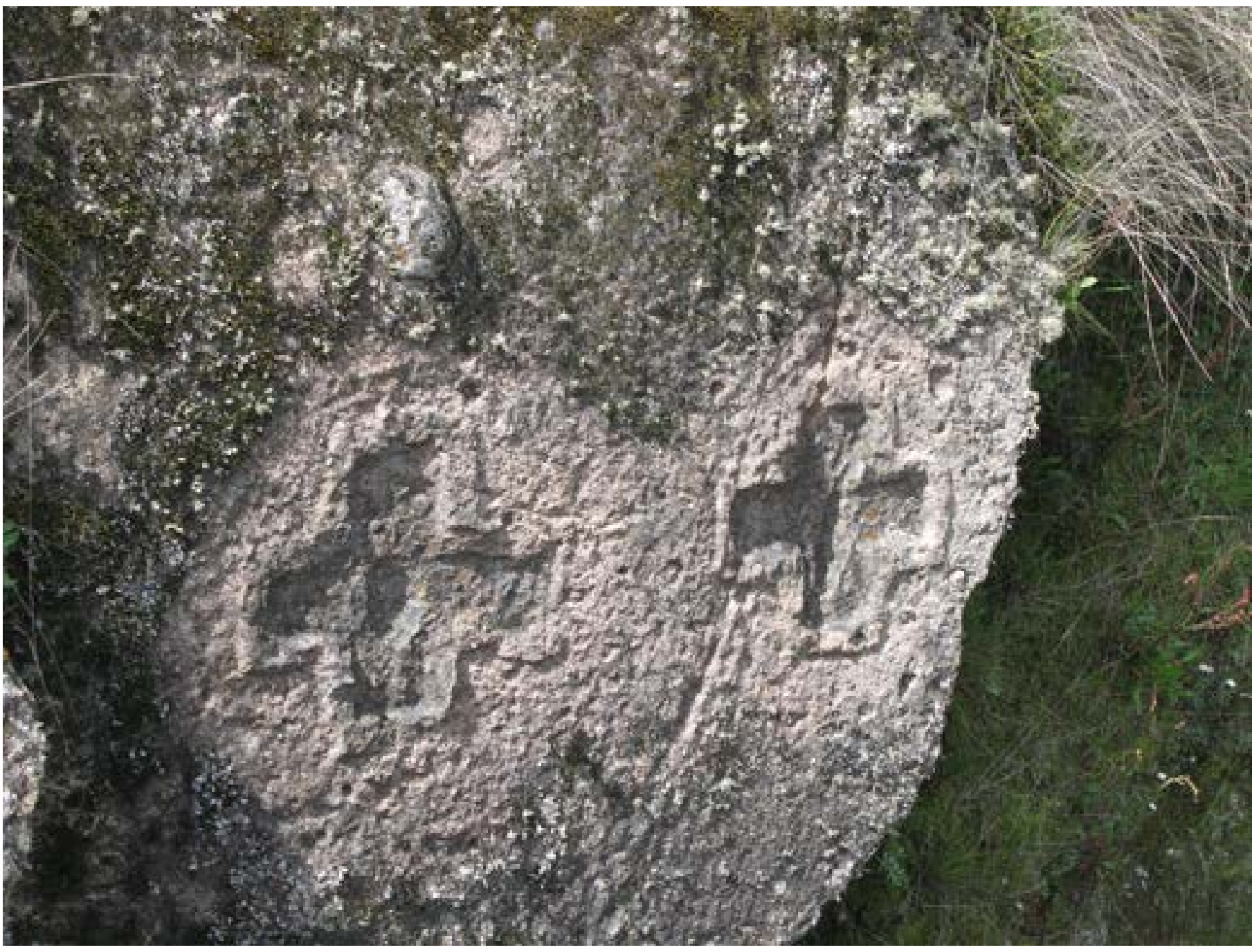

\section{do de abandonado.}

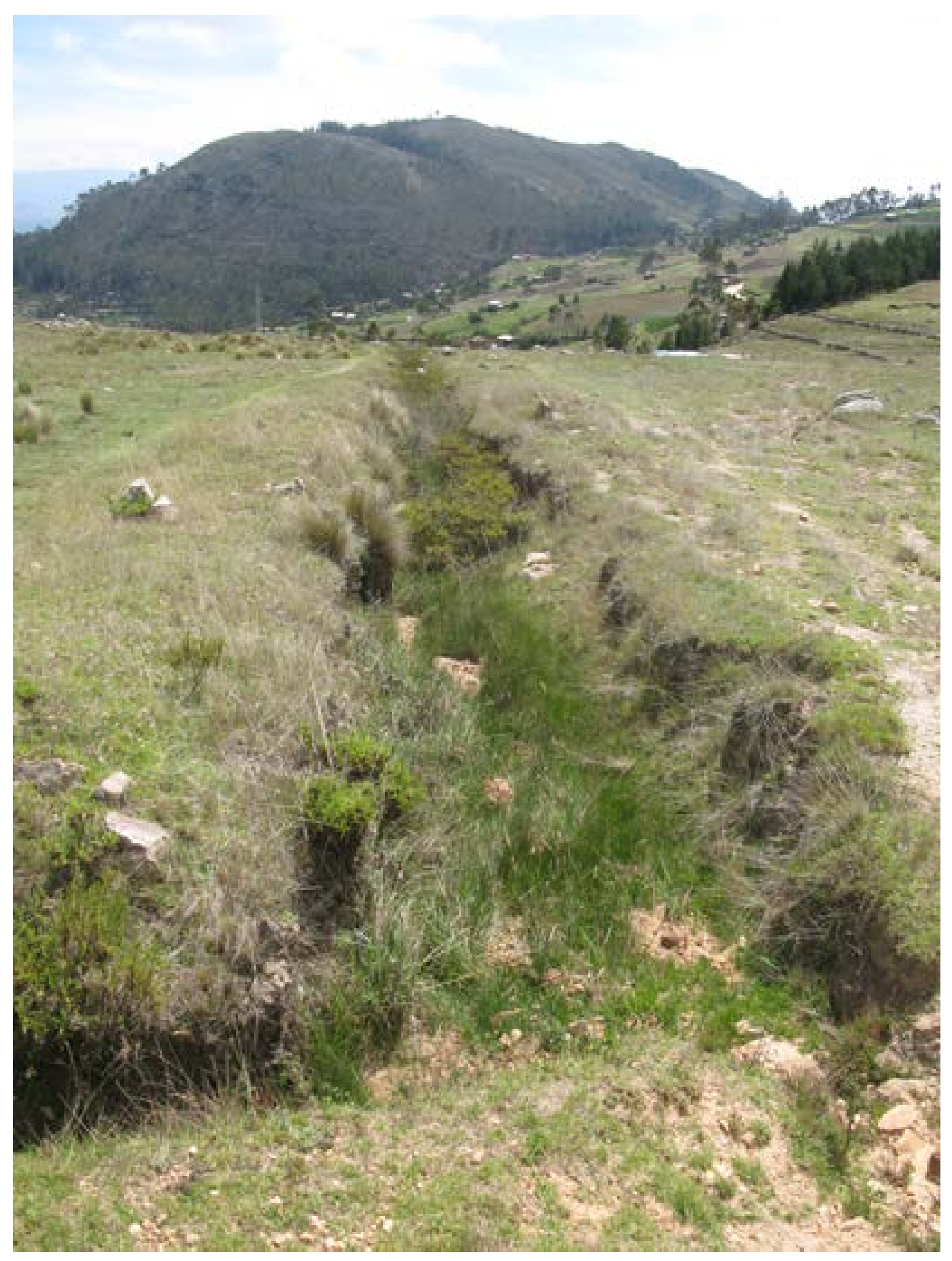

